\title{
THE DYADIC STRUCTURE AND ATOMIC DECOMPOSITION OF $Q$ SPACES IN SEVERAL REAL VARIABLES
}

\author{
Galia Dafni AND Jie XiaO
}

(Received June 10, 2003, revised May 6, 2004)

\begin{abstract}
This paper contains several results relating $Q$ spaces in several real variables with their dyadic counterparts, which are analogues of theorems for BMO and for $Q$ spaces on the circle. In addition, it gives an atomic (or quasi-orthogonal) decomposition for these $Q$ spaces in terms of the same type of atoms used to decompose BMO.
\end{abstract}

1. Introduction. In recent years there has been much interest in a new family of function spaces, called $Q$ spaces. These spaces were originally defined by Aulaskari, Xiao and Zhao in [AXZ] as spaces of holomorphic functions on the unit disk. Following the work of Essén and Xiao [EX] on the boundary values of these functions on the unit circle, the definition was extended to the $n$-dimensional Euclidean space by Essén, Janson, Peng and Xiao in [EJPX].

Fix $\alpha \in(-\infty, \infty)$. For a cube $I$ in $\boldsymbol{R}^{n}$ with sidelength $\ell(I)$, consider the mean quotient of symmetric differences of a function $f \in L^{2}(I)$ as follows:

$$
O_{f, \alpha}(I):=(\ell(I))^{2 \alpha-n} \int_{I} \int_{I} \frac{|f(x)-f(y)|^{2}}{|x-y|^{n+2 \alpha}} d x d y .
$$

We say that $f \in Q_{\alpha}\left(\boldsymbol{R}^{n}\right)$ if $O_{f, \alpha}(I)$ is uniformly bounded, namely

$$
\|f\|_{Q_{\alpha}\left(\boldsymbol{R}^{n}\right)}:=\sup _{I}\left(O_{f, \alpha}(I)\right)^{1 / 2}<\infty,
$$

where the supremum ranges over all cubes $I$ in $\boldsymbol{R}^{n}$ with sides parallel to the coordinate axes. Modulo constants, this defines a norm under which $Q_{\alpha}\left(\boldsymbol{R}^{n}\right)$ becomes a Banach space.

From this definition it is not difficult to see that the spaces $Q_{\alpha}\left(\boldsymbol{R}^{n}\right)$ bear a close connection to the space $\operatorname{BMO}\left(\boldsymbol{R}^{n}\right)$ of functions of bounded mean oscillation, introduced by John and Nirenberg [JN]. Recall that a locally integrable function $f$ belongs to $\operatorname{BMO}\left(\boldsymbol{R}^{n}\right)$ if

$$
\|f\|_{*}:=\sup _{\text {cubes } I} \frac{1}{|I|} \int_{I}|f(x)-f(I)| d x<\infty,
$$

2000 Mathematics Subject Classification. Primary 42B35; Secondary 46E30, 47B38.

Key words and phrases. $Q$ spaces, BMO, dyadic structure, martingales, fractional Carleson measures, atomic decomposition, quasi-orthogonal decomposition.

Project partly supported by the NSERC of Canada and NATEQ. 
where $f(I)$ denotes the mean of $f$ over the cube $I$, i.e. $f(I)=|I|^{-1} \int_{I} f(x) d x$. Equivalently (cf. $[\mathrm{JN}]$ ), one has

$$
\|f\|_{*} \approx \sup _{I}\left(\Phi_{f}(I)\right)^{1 / 2}
$$

with

$$
\Phi_{f}(I):=\frac{1}{|I|} \int_{I}|f(x)-f(I)|^{2} d x .
$$

Rewriting the integral on the right as a double integral gives

$$
\Phi_{f}(I)=\frac{1}{2|I|^{2}} \int_{I} \int_{I}|f(x)-f(y)|^{2} d x d y
$$

and reveals the relationship with $O_{f, \alpha}(I)$ in (1.1).

In fact, the paper [EJPX] showed that

$$
Q_{\alpha}\left(\boldsymbol{R}^{n}\right)=\operatorname{BMO}\left(\boldsymbol{R}^{n}\right) \quad \text { if } \quad \alpha<0,
$$

while

$$
Q_{\alpha}\left(\boldsymbol{R}^{n}\right) \subsetneq \operatorname{BMO}\left(\boldsymbol{R}^{n}\right) \quad \text { if } \quad \alpha \geq 0 .
$$

Furthermore, when $\alpha \geq 1$ (for $n \geq 2$ ) or when $\alpha>1 / 2$ (for $n=1$ ), $Q_{\alpha}\left(\boldsymbol{R}^{n}\right)$ contains only constants. Thus the cases of interest are when $\alpha$ is between 0 and $\min (1, n / 2)$.

It is also important to note that like $\operatorname{BMO}\left(\boldsymbol{R}^{n}\right), Q_{\alpha}\left(\boldsymbol{R}^{n}\right)$ is homogeneous of degree zero, namely:

$$
\|f \circ \phi\|_{Q_{\alpha}\left(\boldsymbol{R}^{n}\right)}=\|f\|_{Q_{\alpha}\left(\boldsymbol{R}^{n}\right)}
$$

for any dilation $\phi(x)=\delta x$ of $\boldsymbol{R}^{n}, \delta>0$. This is in contrast to the case of the homogeneous Sobolev or Besov spaces, whose homogeneity depends on $\alpha$ (see [EJPX] for the relationship between $Q_{\alpha}\left(\boldsymbol{R}^{n}\right)$ and Besov spaces).

The aim of this paper is the further study of $Q_{\alpha}\left(\boldsymbol{R}^{n}\right)$ and its dyadic structure, in particular the analogues for $Q_{\alpha}\left(\boldsymbol{R}^{n}\right)$ of certain well-known results for $\operatorname{BMO}\left(\boldsymbol{R}^{n}\right)$.

In Section 2 we first review some background information on $Q_{\alpha}\left(\boldsymbol{R}^{n}\right)$ from [EJPX]. We then present higher dimensional analogues of some of Janson's results in [Ja] and give a $Q_{\alpha}\left(\boldsymbol{R}^{n}\right)$-version of the main result (concerning the relation between $\operatorname{BMO}\left(\boldsymbol{R}^{n}\right)$ and its dyadic counterpart) of Garnett and Jones [GJ].

In Section 3 we obtain a decomposition of functions in $Q_{\alpha}\left(\boldsymbol{R}^{n}\right)$ into sums of "atoms" of the type used by Uchiyama [U] (following the work of Chang and Fefferman [CF]) to represent BMO $\left(\boldsymbol{R}^{n}\right)$-functions. (See also Rochberg and Semmes [RS] for a different decomposition of $\mathrm{BMO}\left(\boldsymbol{R}^{n}\right)$, and $\mathrm{Wu}$ and Xie [WX] for decomposition theorems for $Q_{p}$ spaces in the unit disk.) The key ingredients in the proof are a quasi-orthogonality lemma and the characterization of $Q_{\alpha}\left(\boldsymbol{R}^{n}\right)$ in terms of fractional Carleson measures, as well as the duality theorem from [DX], identifying $Q_{\alpha}\left(\boldsymbol{R}^{n}\right)$ with the dual of a certain space of distributions, $H H_{-\alpha}^{1}\left(\boldsymbol{R}^{n}\right)$. Thus we may view this decomposition for $Q_{\alpha}\left(\boldsymbol{R}^{n}\right)$ as a kind of dual form of the atomic decomposition of $H_{-\alpha}^{1}\left(\boldsymbol{R}^{n}\right)$ which was proved in [DX].

We would like to thank the referee for the careful reading of our manuscript. 
2. The dyadic structure of $Q_{\alpha}\left(\boldsymbol{R}^{n}\right)$. We first review some notation and then some facts about the dyadic structure of $Q_{\alpha}\left(\boldsymbol{R}^{n}\right)$, which are analogues of similar results for $\operatorname{BMO}\left(\boldsymbol{R}^{n}\right)$.

In the following a cube will always mean a cube in $\boldsymbol{R}^{n}$ with sides parallel to the coordinate axes. We will use the notation $\ell(I)$ for the sidelength of the cube $I,|I|$ for its volume (Lebesgue measure), $\operatorname{diam}(I)$ for its diameter, and $x_{I}$ for its center. For $\delta>0$, we will denote by $\delta I$ the dilated cube, whose center is $x_{I}$ and whose sidelength is $\delta \ell(I)$. Similarly, for $x \in \boldsymbol{R}^{n}, I+x$ will denote the translated cube, namely the cube with center $x_{I}+x$ and sidelength $\ell(I)$.

By $\mathcal{D}_{0}=\mathcal{D}_{0}\left(\boldsymbol{R}^{n}\right)$ we denote the collection of unit cubes whose vertices have integer coordinates, and we set $\mathcal{D}_{k}=\mathcal{D}_{k}\left(\boldsymbol{R}^{n}\right), k \in \boldsymbol{Z}$, to be the collection of all dyadic cubes of sidelength $2^{-k}$, namely all cubes of the form $J=\left\{2^{-k} x ; x \in I\right\}$ for some $I \in \mathcal{D}_{0}$. The collection of all dyadic cubes is then $\mathcal{D}=\bigcup_{-\infty}^{\infty} \mathcal{D}_{k}$. Starting with an arbitrary (not necessarily dyadic) cube $I$, for every $k \geq 0$ we can partition it into $2^{k n}$ subcubes of sidelength $2^{-k} \ell(I)$, forming the collection $\mathcal{D}_{k}(I)$. We write $\mathcal{D}(I)=\bigcup_{0}^{\infty} \mathcal{D}_{k}(I)$.

We use the notation $U \approx V$ to denote the comparability of the quantities $U$ and $V$, i.e., the existence of two positive constants $C_{1}$ and $C_{2}$ satisfying $C_{1} V \leq U \leq C_{2} V$. For convenience, we will always use the letter $C$ to denote a positive constant, which may change from one equation to the next. The constants usually depend on the dimension $n$, and may also depend on $\alpha$ and other fixed parameters.

Now, for $\alpha \in(-\infty, \infty)$ and any cube $I$, let

$$
\Psi_{f, \alpha}(I):=\sum_{k=0}^{\infty} 2^{(2 \alpha-n) k} \sum_{J \in \mathcal{D}_{k}(I)} \Phi_{f}(J),
$$

where $\Phi_{f}$ is as in (1.2), and $\mathcal{D}_{k}(I)$ are the dyadic partitions of $I$ defined above. As shown in [EJPX],

$$
\Phi_{f}(I) \leq \Psi_{f, \alpha}(I) \leq \sum_{k=0}^{\infty} 2^{2 \alpha k} \Phi_{f}(I),
$$

and hence $\Psi_{f, \alpha}(I) \approx \Phi_{f}(I)$ for all $\alpha \in(-\infty, 0)$. Moreover, for $\alpha$ in the positive range we have the following two lemmas (Lemmas 5.8 and 7.7 in [EJPX])

LEMMA 2.1 (EJPX).

(i) Let $\alpha \in(-\infty, 1 / 2)$. Then for any cube I and $f \in L^{2}(I)$,

$$
O_{f, \alpha}(I) \approx \Psi_{f, \alpha}(I) .
$$

(ii) Let $\alpha \in(-\infty, \infty)$. Then for any $f \in L_{\mathrm{loc}}^{2}\left(\boldsymbol{R}^{n}\right)$,

$$
\|f\|_{Q_{\alpha}\left(\boldsymbol{R}^{n}\right)}^{2} \approx \sup _{I} O_{f, \alpha}(I) \approx \sup _{I} \Psi_{f, \alpha}(I),
$$

where the supremum is taken over all cubes in $\boldsymbol{R}^{n}$ with dyadic sidelength. 
Observe that if we replace $f(I)$ in $\Phi_{f}(I)$ with a constant $c_{I}$ depending on the cube $I$, we obtain the following identity $((5.1)$ in $[\mathrm{EJPX}])$ :

$$
|I|^{-1} \int_{I}\left|f(x)-c_{I}\right|^{2} d x=\Phi_{f}(I)+\left|f(I)-c_{I}\right|^{2} .
$$

This implies that $f \in \operatorname{BMO}\left(\boldsymbol{R}^{n}\right)$ if and only if there exist a finite constant $\kappa>0$ and a constant $c_{I}$ for every cube $I \subset \boldsymbol{R}^{n}$ such that

$$
|I|^{-1} \int_{I}\left|f(x)-c_{I}\right|^{2} d x \leq \kappa .
$$

With the help of Lemma 2.1, we can easily obtain a $Q_{\alpha}\left(\boldsymbol{R}^{n}\right)$-version of the last assertion about $\operatorname{BMO}\left(\boldsymbol{R}^{n}\right)$.

THEOREM 2.2. Let $\alpha \in(-\infty, \infty)$ and $f \in L_{\text {loc }}^{2}\left(\boldsymbol{R}^{n}\right)$. Then the following conditions are equivalent :

(i) $f \in Q_{\alpha}\left(\boldsymbol{R}^{n}\right)$.

(ii) There exist a finite constant $\kappa>0$ and a sequence $\left\{c_{J}\right\}_{J \in \mathcal{D}_{k}(I)}$ for every cube $I \subset \boldsymbol{R}^{n}$ and integer $k \geq 0$ such that

$$
\sum_{k=0}^{\infty} 2^{(2 \alpha-n) k} \sum_{J \in \mathcal{D}_{k}(I)}|J|^{-1} \int_{J}\left|f(x)-c_{J}\right|^{2} d x \leq \kappa .
$$

(iii) There exists a finite constant $\kappa>0$ such that for every cube $I \subset \boldsymbol{R}^{n}$,

$$
\sum_{k=0}^{\infty} 2^{(2 \alpha-n) k} \sum_{J \in \mathcal{D}_{k}(I)}|J|^{-1} \int_{0}^{\infty} t m_{f, J}(t) d t \leq \kappa,
$$

where $m_{f, I}(t)=|\{x \in I ;|f(x)-f(I)|>t\}|$.

ProOF. It suffices to show the implication (ii) $\Rightarrow$ (i). If (ii) is true, then for the constant $\kappa$ and sequence $c_{J}$, one has:

$$
\Psi_{f, \alpha}(I) \leq \sum_{k=0}^{\infty} 2^{(2 \alpha-n) k} \sum_{J \in \mathcal{D}_{k}(I)}|J|^{-1} \int_{J}\left|f(x)-c_{J}\right|^{2} d x \leq \kappa,
$$

which implies

$$
\sup _{I} O_{f, \alpha}(I) \leq C \sup _{I} \Psi_{f, \alpha}(I) \leq C \kappa
$$

and hence $f \in Q_{\alpha}\left(\boldsymbol{R}^{n}\right)$, by Lemma 2.1.

Denote by $\delta(\cdot, \cdot)$ the dyadic distance between two points in $\boldsymbol{R}^{n}$ :

$$
\delta(x, y)=\inf \{\ell(I) ; x, y \in I \in \mathcal{D}\} .
$$

It is clear that

$$
|x-y| \leq \sqrt{n} \delta(x, y)
$$


For a cube $I \in \mathcal{D}$ and a function $f \in L^{2}(I)$ let

$$
O_{f, \alpha}^{(d)}(I)=(\ell(I))^{2 \alpha-n} \int_{I} \int_{I} \frac{|f(x)-f(y)|^{2}}{(\delta(x, y))^{n+2 \alpha}} d x d y
$$

be the dyadic fractional mean oscillation (of $f$ on $I$ ). The dyadic version $Q_{\alpha}^{(d)}\left(\boldsymbol{R}^{n}\right)$ of $Q_{\alpha}\left(\boldsymbol{R}^{n}\right)$ is defined as the class of all measurable functions $f$ on $\boldsymbol{R}^{n}$ such that

$$
\|f\|_{Q_{\alpha}^{(d)}\left(\boldsymbol{R}^{n}\right)}:=\sup _{I \in \mathcal{D}}\left(O_{f, \alpha}^{(d)}(I)\right)^{1 / 2}<\infty .
$$

The following basic fact is Lemma 7.1 in $[\mathrm{EJPX}]$ :

LEMMA $2.3(\mathrm{EJPX})$. Let $\alpha \in(-\infty, \infty)$. Then, for any cube $I \in \mathcal{D}$ and $f \in L^{2}(I)$,

$$
\Psi_{f, \alpha}(I) \approx O_{f, \alpha}^{(d)}(I)
$$

This lemma gives immediately that $f \in Q_{\alpha}^{(d)}\left(\boldsymbol{R}^{n}\right)$ if and only if $\sup _{I \in \mathcal{D}} \Psi_{f, \alpha}(I)<\infty$ (see also [EJPX], Theorem 7.2). Moreover we have:

- $Q_{\alpha}^{(d)}\left(\boldsymbol{R}^{n}\right)$ is always a subclass of the dyadic BMO space

$$
\mathrm{BMO}^{(d)}\left(\boldsymbol{R}^{n}\right)=\left\{f \in L_{\mathrm{loc}}^{2}\left(\boldsymbol{R}^{n}\right) ; \sup _{I \in \mathcal{D}} \Phi_{f}(I)<\infty\right\}
$$

- $Q_{\alpha}^{(d)}\left(\boldsymbol{R}^{n}\right)=\mathrm{BMO}^{(d)}\left(\boldsymbol{R}^{n}\right)$ if $\alpha \in(-\infty, 0)$;

- $Q_{\alpha}^{(d)}\left(\boldsymbol{R}^{n}\right)=\boldsymbol{C}$ if $\alpha>n / 2$.

For these see Theorem 7.3 in [EJPX].

Of particular interest is the following identity (see [EJPX], Theorem 7.9):

- $Q_{\alpha}\left(\boldsymbol{R}^{n}\right)=Q_{\alpha}^{(d)}\left(\boldsymbol{R}^{n}\right) \cap \operatorname{BMO}\left(\boldsymbol{R}^{n}\right), \quad \alpha \in(-\infty, 1 / 2)$.

In other words, $Q_{\alpha}\left(\boldsymbol{R}^{n}\right)$ can be characterized by means of $Q_{\alpha}^{(d)}\left(\boldsymbol{R}^{n}\right)$ and $\operatorname{BMO}\left(\boldsymbol{R}^{n}\right)$. Accordingly, in order to study $Q_{\alpha}\left(\boldsymbol{R}^{n}\right)$ it is enough to work with its dyadic counterpart, which is easily understood.

In what follows, we give a characterization of $Q_{\alpha}^{(d)}\left(\boldsymbol{R}^{n}\right)$ in terms of martingales, which is an analogue of a one-dimensional result of Janson ([Ja], Theorem 10). For each $l \in \boldsymbol{Z}$, let $\mathcal{F}_{l}$ be the $\sigma$-field generated by the partition $\mathcal{D}_{l}$. Then to each $f \in L_{\text {loc }}^{1}\left(\boldsymbol{R}^{n}\right)$ we associate the sequence of functions $f_{l}=E\left(f \mid \mathcal{F}_{l}\right)$. In fact, $f_{l}$ is the function that takes the constant value $f(I)$ on each dyadic cube $I \in \mathcal{D}_{l}$.

THEOREM 2.4. Let $\alpha \in(-\infty, \infty)$ and $f \in L_{\text {loc }}^{2}\left(\boldsymbol{R}^{n}\right)$. Then the following conditions are equivalent:

(i) $f \in Q_{\alpha}^{(d)}\left(\boldsymbol{R}^{n}\right)$.

(ii) There exists a finite constant $\kappa>0$ such that for each $l \in \boldsymbol{Z}$,

$$
\sum_{k=0}^{\infty} 2^{2 \alpha k} E\left(\left|f-f_{l+k}\right|^{2} \mid \mathcal{F}_{l}\right) \leq \kappa \quad \text { a.s. }
$$


(iii) There exists a finite constant $\kappa>0$ such that for each $l \in Z$,

$$
\sum_{k=0}^{\infty}\left(\sum_{j=0}^{k} 2^{2 \alpha j}\right) E\left(\left|f_{l+k+1}-f_{l+k}\right|^{2} \mid \mathcal{F}_{l}\right) \leq \kappa \quad \text { a.s. }
$$

Proof. Let $l \in Z$. If $I \in \mathcal{D}_{l}$ and $J \in \mathcal{D}_{k}(I) \subset \mathcal{D}_{l+k}$, then $|J|=2^{-k n}|I|, f(J)=f_{l+k}$ on $J$, and hence

$$
\begin{aligned}
\Psi_{f, \alpha}(I) & =\sum_{k=0}^{\infty} 2^{(2 \alpha-n) k} \sum_{J \in \mathcal{D}_{k}(I)}|J|^{-1} \int_{J}\left|f(x)-f_{l+k}(x)\right|^{2} d x \\
& =\sum_{k=0}^{\infty} 2^{2 \alpha k}|I|^{-1} \int_{I}\left|f(x)-f_{l+k}(x)\right|^{2} d x \\
& =\sum_{k=0}^{\infty} 2^{2 \alpha k} E\left(\left|f-f_{l+k}\right|^{2} \mid \mathcal{F}_{l}\right) .
\end{aligned}
$$

Thus (ii) is equivalent to $\sup _{I \in \mathcal{D}} \Psi_{f, \alpha}(I)<\infty$, which is equivalent to (i) by Lemma 2.3 .

Note that for any nonnegative integer $k$ one has, as in the one-dimensional case:

$$
E\left(\left|f-f_{l+k}\right|^{2} \mid \mathcal{F}_{l}\right)=\sum_{j=k}^{\infty} E\left(\left|f_{l+j+1}-f_{l+j}\right|^{2} \mid \mathcal{F}_{l}\right) .
$$

Inserting this into (2.3) and changing the order of summation, we get:

$$
\sum_{k=0}^{\infty} 2^{2 \alpha k} E\left(\left|f-f_{l+k}\right|^{2} \mid \mathcal{F}_{l}\right)=\sum_{j=0}^{\infty}\left(\sum_{k=0}^{j} 2^{2 \alpha k}\right) E\left(\left|f_{l+j+1}-f_{l+j}\right|^{2} \mid \mathcal{F}_{l}\right),
$$

which implies the equivalence of (ii) and (iii).

Moreover, if $\alpha>0, \sum_{k=0}^{j} 2^{2 \alpha k} \approx 2^{2 \alpha j}$ so (iii) is equivalent to

$$
\sum_{j=0}^{\infty} 2^{2 \alpha j} E\left(\left|f_{l+j+1}-f_{l+j}\right|^{2} \mid \mathcal{F}_{l}\right)<\infty,
$$

which is an exact extension of Theorem 10 in [Ja] to $\boldsymbol{R}^{n}$.

For the case $\alpha<0$, recalling that $Q_{\alpha}^{(d)}\left(\boldsymbol{R}^{n}\right)=\mathrm{BMO}^{(d)}\left(\boldsymbol{R}^{n}\right)$, it follows from Theorem 2.4 that $f \in \mathrm{BMO}^{(d)}\left(\boldsymbol{R}^{n}\right)$ if and only if there is a finite constant $\kappa>0$ such that

$$
E\left(\left|f-f_{j}\right|^{2} \mid \mathcal{F}_{l}\right) \leq \kappa \quad \text { a.s. }
$$

for all $l \in Z$ and all $j \geq l$. (See, for example, remark (b) in Section II.2 of [FeSt].)

Note that $Q_{\alpha}\left(\boldsymbol{R}^{n}\right)$ is translation invariant whereas $Q_{\alpha}^{(d)}\left(\boldsymbol{R}^{n}\right)$ is not. In fact, Theorem 7.8 in [EJPX] states that a function $f$ is in $Q_{\alpha}\left(\boldsymbol{R}^{n}\right)$ if and only if all its translates belong to $Q_{\alpha}^{(d)}\left(\boldsymbol{R}^{n}\right)$. More precisely:

LEMMA 2.5 (EJPX). Let $\alpha \in(-\infty, \infty)$ and let $\tau_{t}$ be the translation operator

$$
\tau_{t} f(x)=f(x-t) \text {. }
$$


Then $f \in Q_{\alpha}\left(\boldsymbol{R}^{n}\right)$ if and only if $\tau_{t} f \in Q_{\alpha}^{(d)}\left(\boldsymbol{R}^{n}\right)$ for all $t \in \boldsymbol{R}^{n}$ with

$$
\sup _{t \in \boldsymbol{R}^{n}}\left\|\tau_{t} f\right\|_{Q_{\alpha}^{(d)}\left(\boldsymbol{R}^{n}\right)}<\infty
$$

This suggests an extension of the general result of Garnett and Jones regarding BMO and $\mathrm{BMO}^{(d)}$ ([GJ], Theorem, p. 352). In order to do so, we need two lemmas. The first one is a generalization of Lemma 5.6 in [EJPX] to the case of an arbitrary number of cubes.

LEMMA 2.6. Let $\alpha \in(-\infty, 1 / 2)$. If a cube I is contained in the union of $l$ cubes of the same size, namely $I \subset I^{1} \cup \cdots \cup I^{l}$, with $\left|I^{j}\right|=|I|$ for $1 \leq j \leq l$, then

$$
\Phi_{f}(I) \leq \sum_{j=1}^{l} \Phi_{f}\left(I^{j}\right)+\frac{2(l-1)}{l^{2}} \sum_{1 \leq i<j \leq l}\left|f\left(I^{i}\right)-f\left(I^{j}\right)\right|^{2}
$$

and

$$
\Psi_{f, \alpha}(I) \leq C_{l}\left(\sum_{j=1}^{l} \Psi_{f, \alpha}\left(I^{j}\right)+\sum_{1 \leq i<j \leq l}\left|f\left(I^{i}\right)-f\left(I^{j}\right)\right|^{2}\right) .
$$

ProOF. The proof is similar to that of Lemma 5.6 in [EJPX]. First, using identity (2.2) with the constant $c_{I}=c:=l^{-1} \sum_{i=1}^{l} f\left(I^{i}\right)$, we have

$$
\begin{aligned}
\Phi_{f}(I) & \leq|I|^{-1} \int_{I}|f(x)-c|^{2} d x \leq \sum_{j=1}^{l}\left|I^{j}\right|^{-1} \int_{I^{j}}|f(x)-c|^{2} d x \\
& =\sum_{j=1}^{l}\left\{\Phi_{f}\left(I^{j}\right)+\left|f\left(I^{j}\right)-c\right|^{2}\right\} \\
& \leq \sum_{j=1}^{l}\left\{\Phi_{f}\left(I^{j}\right)+\left(l^{-1} \sum_{i=1}^{l}\left|f\left(I^{j}\right)-f\left(I^{i}\right)\right|\right)^{2}\right\} \\
& \leq \sum_{j=1}^{l}\left\{\Phi_{f}\left(I^{j}\right)+\frac{(l-1)}{l^{2}} \sum_{i \neq j}\left|f\left(I^{i}\right)-f\left(I^{j}\right)\right|^{2}\right\} \\
& =\sum_{j=1}^{l} \Phi_{f}\left(I^{j}\right)+\frac{2(l-1)}{l^{2}} \sum_{1 \leq i<j \leq l}\left|f\left(I^{i}\right)-f\left(I^{j}\right)\right|^{2} .
\end{aligned}
$$

For (2.5) we can, as is done in [EJPX], assume by homogeneity that $I=I^{0}+\mathbf{x}$, with $I^{0}=[0,1]^{n}$ and $\mathbf{x}=x^{1} \mathbf{e}^{1}+\cdots+x^{n} \mathbf{e}^{n}$, where $x^{i} \in[0,1)$ and $\mathbf{e}^{i}$ is the unit vector in the $i$ th coordinate direction. Then each $I^{j}$ in the statement of the lemma can be assumed to be of the form $I^{j}=I^{0}+\mathbf{v}^{j}$, where $\mathbf{v}^{j}$ is a vector whose coordinates are 0's and 1's. There are at most $2^{n}$ such choices, and any $I^{j}$ which is not of this form would be superfluous and would just add to the right-hand-side of (2.5). Note that if $x^{i}=0$, we need only consider those $I^{j}$ 's for which the vector $\mathbf{v}^{j}$ has a zero in the $i$ th coordinate. In [EJPX] it was assumed $x^{2}=\cdots=x^{n}=0$ and there were only two cubes containing $I$ (i.e. $l=2$ ). 
Now suppose $k$ is a nonnegative integer. Following the notation in [EJPX], we denote by $\mathcal{D}_{k}^{*}$ the union $\bigcup_{j=1}^{l} \mathcal{D}_{k}\left(I^{j}\right)$ of the $l 2^{n k}$ dyadic cubes of sidelength $2^{-k}$ contained in $I^{1} \cup \cdots \cup$ $I^{l}$. If $I_{k} \in \mathcal{D}_{k}(I)$, then

$$
I_{k}=\left[0,2^{-k}\right]^{n}+\mathbf{x}+t_{k}^{1} \mathbf{e}^{1}+\cdots t_{k}^{n} \mathbf{e}^{n},
$$

where $t_{k}^{j}$ is a number of the from $m 2^{-k}, m$ an integer, $0 \leq m<2^{k}$. Now the cube $I_{k}^{0}=$ $\left[0,2^{-k}\right]^{n}+t_{k}^{1} \mathbf{e}^{1}+\cdots t_{k}^{n} \mathbf{e}^{n}$ is in $\mathcal{D}_{k}\left(I^{0}\right)$, so $I_{k}=I_{k}^{0}+\mathbf{x}$ is contained in $l$ dyadic cubes $I_{k}^{1} \cup \cdots \cup I_{k}^{l}$, each of which is in $\mathcal{D}_{k}^{*}$. Here $l$ is the same number of cubes as in the statement of the lemma. In fact, if the point $\mathbf{x}+t_{k}^{1} \mathbf{e}^{1}+\cdots t_{k}^{n} \mathbf{e}^{n}$ belongs to the cube $I_{k}^{1} \in \mathcal{D}_{k}^{*}$, then the other cubes are given by $I_{k}^{j}=I_{k}^{1}+2^{-k} \mathbf{v}^{j}$, where the vectors $\mathbf{v}^{j}, 1 \leq j \leq l$ are as above (depending on the number of nonzero coordinates $x^{i}$ of $\mathbf{x}$ ). Note that if $x^{i}$ is an integer multiple of $2^{-k}$, it is possible that $I_{k}$ is contained in less than $l$ cubes, but we can always use $l$ cubes.

Applying (2.4) to $I_{k}$, we get

$$
\begin{aligned}
\Phi_{f}\left(I_{k}\right) & \leq \sum_{j=1}^{l} \Phi_{f}\left(I_{k}^{j}\right)+\frac{2(l-1)}{l^{2}} \sum_{1 \leq i<j \leq l}\left|f\left(I_{k}^{i}\right)-f\left(I_{k}^{j}\right)\right|^{2} \\
& =\sum_{j=1}^{l} \Phi_{f}\left(I_{k}^{j}\right)+\frac{2(l-1)}{l^{2}} \sum_{1 \leq i<j \leq l}\left|f\left(I_{k}^{1}+2^{-k} \mathbf{v}^{i}\right)-f\left(I_{k}^{1}+2^{-k} \mathbf{v}^{j}\right)\right|^{2} .
\end{aligned}
$$

Again following [EJPX], let us denote by $\mathcal{D}_{k}^{0}$ the set of $J \in \mathcal{D}_{k}^{*}$ such that $J+2^{-k} \mathbf{v}^{j} \in \mathcal{D}_{k}^{*}$ for $j=1, \ldots, l$. Since different $I_{k}$ in $\mathcal{D}_{k}(I)$ corresponds to different $I_{k}^{1}$ in $\mathcal{D}_{k}^{0}$, if we sum over all $I_{k} \in \mathcal{D}_{k}(I)$ and over all $k \geq 0$, we get

$$
\begin{aligned}
\Psi_{f, \alpha}(I)= & \sum_{k=0}^{\infty} \sum_{I_{k} \in \mathcal{D}_{k}(I)} 2^{(2 \alpha-n) k} \Phi_{f}\left(I_{k}\right) \\
\leq & l \sum_{k=0}^{\infty} 2^{(2 \alpha-n) k} \sum_{J \in \mathcal{D}_{k}^{*}} \Phi_{f}(J) \\
& +\frac{2(l-1)}{l^{2}} \sum_{k=0}^{\infty} 2^{(2 \alpha-n) k} \sum_{J \in \mathcal{D}_{k}^{0}} \sum_{1 \leq i<j \leq l}\left|f\left(J+2^{-k} \mathbf{v}^{i}\right)-f\left(J+2^{-k} \mathbf{v}^{j}\right)\right|^{2} \\
\leq & l \sum_{j=1}^{l} \Psi_{f, \alpha}\left(I^{j}\right) \\
& +\frac{2(l-1)}{l^{2}} \sum_{k=0}^{\infty} 2^{(2 \alpha-n) k} \sum_{J \in \mathcal{D}_{k}^{0}} \sum_{1 \leq i<j \leq l}\left|f\left(J+2^{-k} \mathbf{v}^{i}\right)-f\left(J+2^{-k} \mathbf{v}^{j}\right)\right|^{2} .
\end{aligned}
$$

Continuing as in [EJPX], fix $k \geq 0$, a cube $J \in \mathcal{D}_{k}^{0}$, and vectors $\mathbf{v}^{i}$ and $\mathbf{v}^{j}$. For simplicity, write $J_{0}=J+2^{-k} \mathbf{v}^{i}, K_{0}=J+2^{-k} \mathbf{v}^{j}$. Then as in [EJPX] we have two sequences of dyadic cubes $J_{0} \subset J_{1} \subset \cdots \subset J_{m}$ and $K_{0} \subset K_{1} \subset \cdots \subset K_{m}$ with sidelengths $\ell\left(J_{r}\right)=2^{-k+r}$ and 
$J_{m}=K_{m}$ being the smallest dyadic cube containing $J_{0}$ and $K_{0}$. Thus we can repeat estimate (5.14) in [EJPX], using Cauchy-Schwarz and the fact that $\left|f\left(J_{r}\right)-f\left(J_{r-1}\right)\right|^{2} \leq 2^{n} \Phi_{f}\left(J_{r}\right)$ :

$$
\left|f\left(J_{0}\right)-f\left(K_{0}\right)\right|^{2} \leq C \sum_{r=1}^{m} r^{2}\left(\Phi_{f}\left(J_{r}\right)+\Phi_{f}\left(K_{r}\right)\right) .
$$

Note that since $I^{1} \cup \cdots \cup I^{l} \subset[0,2]^{n}$, we must have $m \leq k+1$. Moreover, in the case $m=k+1, J_{k}$ and $K_{k}$ must be $I^{i}$ and $I^{j}$ for some $i, j, 1 \leq i<j \leq l$. In this case we end up with the following analogue of estimate (5.15) in [EJPX]:

$$
\left|f\left(J_{0}\right)-f\left(K_{0}\right)\right|^{2} \leq C \sum_{r=1}^{k} r^{2}\left(\Phi_{f}\left(J_{r}\right)+\Phi_{f}\left(K_{r}\right)\right)+C\left|f\left(I^{i}\right)-f\left(I^{j}\right)\right|^{2} .
$$

Now we need to sum over all $J \in \mathcal{D}_{k}^{0}$ and all choices of $J_{0}=J+2^{-k} \mathbf{v}^{i}$ and $K_{0}=$ $J+2^{-k} \mathbf{v}^{j}, 1 \leq i<j \leq l$. As pointed out in [EJPX], the cubes $J_{r}$ and $K_{r}$ in (2.7) belong to $\mathcal{D}_{k-r}^{*}, 1 \leq r \leq k$. Conversely, each cube $J^{\prime} \in \mathcal{D}_{k-r}^{*}$ corresponds to a $J_{r}$ or $K_{r}$ only for those $J_{0}$ and $K_{0}$ which lie adjacent to the boundaries of its $2^{n}$ dyadic subcubes of sidelength $2^{-k+r-1}$ (otherwise both $J_{0}$ and $K_{0}$ would lie in a dyadic cube of sidelength $2^{-k+r-1}$, thereby making $m \leq r-1)$. Thus there are at most $4 n 2^{(n-1) r}$ choices of $J_{0}$ or $K_{0}$ corresponding to $J^{\prime} \in \mathcal{D}_{k-r}^{*}$. For the case $m=k+1$, in which we apply (2.8), each cube $I^{j}, 1 \leq j \leq l$, can appear as $J_{k}$ or $K_{k}$ for at most $4 n 2^{(n-1) k}$ choices of $J_{0}$ or $K_{0}$. Thus we get

$$
\begin{aligned}
& \sum_{J \in \mathcal{D}_{k}^{0}} \sum_{1 \leq i<j \leq l}\left|f\left(J+2^{-k} \mathbf{v}^{i}\right)-f\left(J+2^{-k} \mathbf{v}^{j}\right)\right|^{2} \\
& \quad \leq C \sum_{r=1}^{k} \sum_{J^{\prime} \in \mathcal{D}_{k-r}^{*}} r^{2} 2^{(n-1) r} \Phi_{f}\left(J^{\prime}\right)+C 2^{(n-1) k} \sum_{1 \leq i<j \leq l}\left|f\left(I^{i}\right)-f\left(I^{j}\right)\right|^{2} .
\end{aligned}
$$

Finally, summing over all $k \geq 0$ and letting $s=k-r$, we have

$$
\begin{aligned}
\sum_{k=0}^{\infty} 2^{(2 \alpha-n) k} \sum_{J \in \mathcal{D}_{k}^{0}} \sum_{1 \leq i<j \leq l}\left|f\left(J+2^{-k} \mathbf{v}^{i}\right)-f\left(J+2^{-k} \mathbf{v}^{j}\right)\right|^{2} \\
\leq C \sum_{k=0}^{\infty} 2^{(2 \alpha-n) k}\left\{\sum_{r=1}^{k} \sum_{J^{\prime} \in \mathcal{D}_{k-r}^{*}} r^{2} 2^{(n-1) r} \Phi_{f}\left(J^{\prime}\right)+C 2^{(n-1) k} \sum_{1 \leq i<j \leq l}\left|f\left(I^{i}\right)-f\left(I^{j}\right)\right|^{2}\right\} \\
=C \sum_{r=0}^{\infty} \sum_{s=0}^{\infty} \sum_{J^{\prime} \in \mathcal{D}_{s}^{*}} r^{2} 2^{(n-1) r+(2 \alpha-n)(r+s)} \Phi_{f}\left(J^{\prime}\right) \\
\quad+C \sum_{k=0}^{\infty} 2^{(2 \alpha-1) k} \sum_{1 \leq i<j \leq l}\left|f\left(I^{i}\right)-f\left(I^{j}\right)\right|^{2} \\
=C \sum_{r=0}^{\infty} r^{2} 2^{(2 \alpha-1) r} \sum_{1 \leq j \leq l}^{\infty} \sum_{s=0}^{\infty} \sum_{J^{\prime} \in \mathcal{D}_{s}\left(I^{j}\right)} 2^{(2 \alpha-n) s} \Phi_{f}\left(J^{\prime}\right)
\end{aligned}
$$




$$
\begin{aligned}
& +C \sum_{k=0}^{\infty} 2^{(2 \alpha-1) k} \sum_{1 \leq i<j \leq l}\left|f\left(I^{i}\right)-f\left(I^{j}\right)\right|^{2} \\
\leq & C \sum_{1 \leq j \leq l} \Psi_{f, \alpha}\left(I^{j}\right)+C \sum_{1 \leq i<j \leq l}\left|f\left(I^{i}\right)-f\left(I^{j}\right)\right|^{2},
\end{aligned}
$$

since $\alpha<1 / 2$ implies that $\sum_{k=0}^{\infty} 2^{(2 \alpha-1) k}$ and $\sum_{r=0}^{\infty} r^{2} 2^{(2 \alpha-1) r}$ are finite. Inserting this into (2.6), we get (2.5). Note that the constant $C_{l}$ appearing in (2.5) can be replaced by a constant depending only on the dimension $n$ and $\alpha$, since, as explained above, we can assume $l \leq 2^{n}$.

The second lemma required is an extension of Lemma 6 in [Ja] to $\boldsymbol{R}^{n}$.

Lemma 2.7. Suppose $J$ is a fixed dyadic cube in $\boldsymbol{R}^{n}$, with sidelength $2^{K}$ for some $K \in \boldsymbol{Z}$. Let $I \subset \boldsymbol{R}^{n}$ be a cube (not necessarily dyadic) of sidelength $2^{k}, k \in \boldsymbol{Z}$, and suppose $t \in \boldsymbol{R}^{n}$. Consider the intersection $(I+t) \cap J$ of the translated cube $I+t$ with the fixed cube $J$. Let $I_{t}^{\prime}$ be the smallest dyadic cube of sidelength at least $2^{\min \{k, K\}}$ which contains $(I+t) \cap J$, and let $\ell\left(I_{t}^{\prime}\right)=2^{L}$. Define $m_{J}(I, t)$ as follows:

(i) if $|(I+t) \cap J|=0$, $\operatorname{set}_{J}(I, t)=0$;

(ii) if $|(I+t) \backslash J|=0($ i.e. $(I+t) \subset J)$, set $m_{J}(I, t)=L-k$;

(iii) if $|(I+t) \backslash J|>0$ and $|(I+t) \cap J|>0$, set $m_{J}(I, t)=\max \{0, K-k\}$.

Then for every nonnegative integer $M$,

$$
\left|\left\{t \in \boldsymbol{R}^{n}: m_{J}(I, t)>M\right\}\right| \leq 2^{(K+1) n-M},
$$

and for $p \in(0, \infty)$,

$$
\int_{\boldsymbol{R}^{n}}\left(m_{J}(I, t)\right)^{p} d t \leq 2^{(K+1) n+1} \sum_{l=1}^{\infty} l^{p} 2^{-l} \leq C_{n, p}|J|<\infty .
$$

Proof. By definition, $m_{J}(I, t) \geq 0$ in all cases. Moreover, the cube $I_{t}^{\prime} \subset J$ implies that $L \leq K$ and therefore $m_{J}(I, t) \leq \max \{0, K-k\}$ in all cases. If $k \geq K$, then $m_{J}(I, t)$ is identically 0 and there is nothing to prove. Thus we may assume $k<K$, and prove (2.9) for integers $M$ with $0 \leq M<K-k$.

Since (2.9) is translation invariant in $I$, we can fix $J=\left[0,2^{K}\right]^{n}$, and assume $I=$ $\left[0,2^{k}\right]^{n}$. Observe that $m_{J}(I, t)>M$ is equivalent to the fact that $I+t$ intersects $J$ (nontrivially) but every dyadic subcube $J^{\prime} \subset J$ with sidelength $\ell\left(J^{\prime}\right)=2^{k+M}$ does not contain $I+t$. Thus for each such dyadic subcube $J^{\prime}$ we get a set of vectors

$$
S_{J^{\prime}}=\left\{t \in J^{\prime} ; I+t \text { is not contained in } J^{\prime}\right\}
$$

with volume $\left|S_{J^{\prime}}\right|$ equal to $2^{(k+M) n}-\left(2^{k+M}-2^{k}\right)^{n}$. In addition, we have the set

$$
S_{0}=\left\{t \in \boldsymbol{R}^{n} ;|(I+t) \cap J|>0,|I+t \backslash J|>0\right\}
$$


with volume $\left|S_{0}\right|$ equal to $\left(2^{K}+2^{k}\right)^{n}-2^{K n}$. Thus

$$
\begin{aligned}
\left|\left\{t \in \boldsymbol{R}^{n}: m_{J}(I, t)>M\right\}\right| & =\sum_{J^{\prime} \in \mathcal{D}_{K-k-M}(J)}\left|S_{J^{\prime}}\right|+\left|S_{0}\right| \\
& =2^{(K-k-M) n}\left(2^{(k+M) n}-\left(2^{k+M}-2^{k}\right)^{n}\right)+\left(2^{K}+2^{k}\right)^{n}-2^{K n} \\
& =2^{K n}\left[1-\left(1-2^{-M}\right)^{n}\right]+2^{K n}\left[\left(1+2^{k-K}\right)^{n}-1\right] \\
& \leq 2^{K n}\left[n 2^{-M}+\left(2^{n}-1\right) 2^{k-K}\right] \\
& \leq 2^{(K+1) n-M},
\end{aligned}
$$

and (2.9) holds.

Now for $p>0$ one has:

$$
\begin{aligned}
\int_{\boldsymbol{R}^{n}}\left(m_{J}(I, t)\right)^{p} d t & =\sum_{l=0}^{K-k} l^{p}\left|\left\{t \in \boldsymbol{R}^{n}: m_{J}(I, t)=l\right\}\right| \\
& \leq \sum_{l=1}^{K-k} l^{p}\left|\left\{t \in \boldsymbol{R}^{n}: m_{J}(I, t)>l-1\right\}\right| \\
& \leq 2^{(K+1) n+1} \sum_{l=1}^{\infty} l^{p} 2^{-l},
\end{aligned}
$$

proving (2.10).

With these two lemmas we have the following analogue of the result of Garnett and Jones (see also Theorem 5 in [Ja] for a one-dimensional $Q_{p}$ version):

THEOREM 2.8. Let $\alpha \in(-\infty, 1 / 2)$. Suppose $F$ is a function on $\boldsymbol{R}^{n} \times \boldsymbol{R}^{n}$ such that for each $t \in \boldsymbol{R}^{n}, F(t, \cdot) \in Q_{\alpha}^{(d)}\left(\boldsymbol{R}^{n}\right)$ with support in a fixed dyadic cube, and $\int_{\boldsymbol{R}^{n}} F(t, x) d x=0$. Moreover, assume $\|F(t, \cdot)\|_{Q_{\alpha}^{(d)}\left(\boldsymbol{R}^{n}\right)}$ is essentially bounded as a function of $t$. Then for every $N \in(0, \infty)$, the averaging function $f_{N}$ belongs to $Q_{\alpha}\left(\boldsymbol{R}^{n}\right)$, where $f_{N}$ is defined on $\boldsymbol{R}^{n}$ by

$$
f_{N}(x)=\frac{1}{(2 N)^{n}} \int_{[-N, N]^{n}} F(t, x+t) d t .
$$

ProOF. We proceed by analogy with Janson's proof in the case of the circle (see [Ja]). Set $f_{t}(x)=F(t, x)$ and $h_{t}(x)=F(t, x+t)$. Let $J$ denote the fixed dyadic cube which contains the support of $f_{t}$ for every $t$. Assume that $I$ is a cube in $\boldsymbol{R}^{n}$ (not necessarily dyadic) of sidelength $2^{k}, k \in \boldsymbol{Z}$. Consider the translate $I+t$ for some $t \in[-N, N]^{n}$. We want to estimate $\Psi_{h_{t}, \alpha}(I)=\Psi_{f_{t}, \alpha}(I+t)$ in terms of $\left\|f_{t}\right\|_{Q_{\alpha}^{(d)}\left(\boldsymbol{R}^{n}\right)}$. More specifically, we want to prove the estimate

$$
\left(\Psi_{f_{t}, \alpha}(I+t)\right)^{1 / 2} \leq C\left(m_{J}(I, t)+1\right)\left\|f_{t}\right\|_{Q_{\alpha}^{(d)}\left(\boldsymbol{R}^{n}\right)},
$$

where $m_{J}(I, t)$ is the integer defined in Lemma 2.7. There are several cases depending on the nature of the intersection $(I+t) \cap J$. 
The trivial case is when the measure $|(I+t) \cap J|=0$, in which case Lemma 2.7 defines $m_{J}(I, t)$ to be zero, and on the other hand $f_{t}=0$ almost everywhere on $I+t$ gives $\Psi_{f_{t}, \alpha}(I+t)=0$.

The next case is that in which $|(I+t) \backslash J|=0$, or more simply (if we consider closed cubes) $I+t \subset J$. Then $I+t$ is contained in the union of at most $2^{n}$ adjacent dyadic cubes of equal sidelength, namely $I+t \subset I_{1} \cup \cdots \cup I_{l}$, with $\ell\left(I_{j}\right)=\ell(I)=2^{k}$ for $1 \leq j \leq l$, $I_{j}$ dyadic, and $l \leq 2^{n}$. Moreover, if $I_{t}^{\prime}$ is the smallest dyadic cube containing $I+t$, then $I_{1} \cup \cdots \cup I_{l} \subset I_{t}^{\prime}$.

By Lemma 2.6,

$$
\begin{aligned}
\left(\Psi_{f_{t}, \alpha}(I+t)\right)^{1 / 2} & \leq C_{l}\left(\sum_{j=1}^{l}\left(\Psi_{f_{t}, \alpha}\left(I_{j}\right)\right)^{1 / 2}+\sum_{1 \leq j<k \leq l}\left|f_{t}\left(I_{j}\right)-f_{t}\left(I_{k}\right)\right|\right) \\
& \leq C\left\|f_{t}\right\|_{Q_{\alpha}^{(d)}\left(\boldsymbol{R}^{n}\right)}+C \sum_{1 \leq j<k \leq l}\left|f_{t}\left(I_{j}\right)-f_{t}\left(I_{k}\right)\right|,
\end{aligned}
$$

where the constants depend only on $\alpha$ and $n$ (since $l \leq 2^{n}$ ). For each cube $I_{j}$, consider the sequence of dyadic cubes $I_{j}=J_{j, 0} \subset J_{j, 1} \subset \cdots \subset J_{j, m}=I_{t}^{\prime}$ with $\ell\left(J_{j, i+1}\right)=2 \ell\left(J_{j, i}\right)$. Here $m=L-k$, where $\ell\left(I_{t}^{\prime}\right)=2^{L}$, and this is exactly the integer $m_{J}(I, t)$ defined by Lemma 2.7 in this case. Recall (see (5.4) in [EJPX]) that if $I \subset J$ then $|f(I)-f(J)|^{2} \leq$ $(|J| /|I|) \Phi_{f}(J)$. Thus

$$
\begin{aligned}
\left|f_{t}\left(I_{j}\right)-f_{t}\left(I_{k}\right)\right| & \leq \sum_{i=1}^{m}\left|f_{t}\left(J_{j, i}\right)-f_{t}\left(J_{j, i-1}\right)\right|+\sum_{i=1}^{m}\left|f_{t}\left(J_{k, i}\right)-f_{t}\left(J_{k, i-1}\right)\right| \\
& \leq 2^{n / 2} \sum_{i=1}^{m}\left(\Phi_{f_{t}}\left(J_{j, i}\right)\right)^{1 / 2}+2^{n / 2} \sum_{i=1}^{m}\left(\Phi_{f_{t}}\left(J_{k, i}\right)\right)^{1 / 2} \\
& \leq 2^{n / 2+1} m_{J}(I, t)\left\|f_{t}\right\|_{Q_{\alpha}^{(d)}\left(\boldsymbol{R}^{n}\right)} .
\end{aligned}
$$

Inserting this in (2.12), we get (2.11).

The remaining case is that in which $I+t$ intersects both $J$ and its complement, i.e. $|(I+t) \backslash J|>0$ and $|(I+t) \cap J|>0$. Again write $I+t \subset I_{1} \cup \cdots \cup I_{l}$, with $\ell\left(I_{j}\right)=\ell(I)=2^{k}$ for $1 \leq j \leq l, I_{j}$ dyadic, and $l \leq 2^{n}$, and use Lemma 2.6 to get inequality (2.12).

Now for each pair $j, k$, we have three cases. If both $I_{j}$ and $I_{k}$ have interiors which are disjoint from $J$, then $f_{t}\left(I_{j}\right)=f_{t}\left(I_{k}\right)=0$. If both $I_{j}$ and $I_{k}$ are contained in $J$, we proceed as above, using the fact that in this case $k \leq K$, where $\ell(J)=2^{K}$, and $m_{J}(I, t)=K-k$, so if $L$ is as above (i.e. $\left.\ell\left(I_{t}^{\prime}\right)=2^{L}\right)$, then $L-k \leq m_{J}(I, t)$. Thus we again get

$$
\left|f_{t}\left(I_{j}\right)-f_{t}\left(I_{k}\right)\right| \leq 2^{n / 2+1} m_{J}(I, t)\left\|f_{t}\right\|_{Q_{\alpha}^{(d)}\left(\boldsymbol{R}^{n}\right)} .
$$

Otherwise, one of these two cubes, say $I_{k}$, must have disjoint interior with $J$, while the other, $I_{j}$, must either contain or be contained in $J$. Since $f_{t}$ is supported in $J$, this gives $f_{t}\left(I_{k}\right)=0$ and $\left|f_{t}\left(I_{j}\right)-f_{t}\left(I_{k}\right)\right|=\left|f_{t}\left(I_{j}\right)\right|$.

If $I_{j} \supset J$, then $\int_{I_{j}} f_{t}(x) d x=\int_{J} f_{t}(x) d x=\int_{\boldsymbol{R}^{n}} f_{t}(x) d x=0$ so $f_{t}\left(I_{j}\right)=0$ and $\left|f_{t}\left(I_{j}\right)-f_{t}\left(I_{k}\right)\right|=0=m_{J}(I, t)$ (since $k \geq K$ ). Otherwise $I_{j} \subset J$, hence we let $J_{j, i}$ be the 
sequence of dyadic cubes with $I_{j}=J_{j, 0} \subset J_{j, 1} \subset \cdots \subset J_{j, m}=J$, where again in this case $m=m_{J}(I, t)=K-k$, and $\ell\left(J_{j, i+1}\right)=2 \ell\left(J_{j, i}\right)$. Thus we can write

$$
\begin{aligned}
\left|f_{t}\left(I_{j}\right)\right| & =\left|f_{t}\left(I_{j}\right)-f_{t}(J)\right| \leq \sum_{i=1}^{m}\left|f_{t}\left(J_{j, i}\right)-f_{t}\left(J_{j, i-1}\right)\right| \leq 2^{n / 2} \sum_{i=1}^{m}\left(\Phi_{f_{t}}\left(J_{j, i}\right)\right)^{1 / 2} \\
& \leq 2^{n / 2} m_{J}(I, t)\left\|f_{t}\right\|_{Q_{\alpha}^{(d)}(\boldsymbol{R})} .
\end{aligned}
$$

Inserting the estimates for both cases into (2.12), we again get (2.11).

Finally, in order to estimate $\left(\Psi_{f_{N}, \alpha}(I)\right)^{1 / 2}$, we note that $\left(\Psi_{f, \alpha}(I)\right)^{1 / 2}$ may be regarded as a weighted $L^{2}$ norm on $I \times I$ by writing (see [Ja], (10)):

$$
\begin{aligned}
\Psi_{f, \alpha}(I) & =\sum_{k=0}^{\infty} 2^{(2 \alpha-n) k} \sum_{J \in \mathcal{D}_{k}(I)} \Phi_{f}(J) \\
& =\sum_{k=0}^{\infty} 2^{(2 \alpha-n) k-1} \sum_{J \in \mathcal{D}_{k}(I)}|J|^{-2} \int_{J} \int_{J}|f(x)-f(y)|^{2} d x d y \\
& =\int_{I} \int_{I}|f(x)-f(y)|^{2}\left(|I|^{-2} \sum_{k=0}^{\infty} \sum_{J \in \mathcal{D}_{k}(I)} 2^{(2 \alpha+n) k-1} \chi_{J}(x) \chi_{J}(y)\right) d x d y .
\end{aligned}
$$

Applying Minkowski's integral inequality to

$$
f_{N}=(2 N)^{-n} \int_{[-N, N]^{n}} h_{t} d t
$$

we get

$$
\begin{aligned}
\left(\Psi_{f_{N}, \alpha}(I)\right)^{1 / 2} & \leq(2 N)^{-n} \int_{[-N, N]^{n}}\left(\Psi_{h_{t}, \alpha}(I)\right)^{1 / 2} d t \\
& \leq C(2 N)^{-n} \int_{[-N, N]^{n}}\left(m_{J}(I, t)+1\right)\left\|f_{t}\right\|_{Q_{\alpha}^{(d)}\left(\boldsymbol{R}^{n}\right)} d t \\
& \leq C \sup _{t}\|F(t, \cdot)\|_{Q_{\alpha}^{(d)}\left(\boldsymbol{R}^{n}\right)}\left(1+(2 N)^{-n} \int_{t \in \boldsymbol{R}^{n}} m_{J}(I, t) d t\right) \\
& \leq C \sup _{t}\|F(t, \cdot)\|_{Q_{\alpha}^{(d)}\left(\boldsymbol{R}^{n}\right)}\left(1+(2 N)^{-n}|J|\right)
\end{aligned}
$$

by (2.10) in Lemma 2.7. This shows that $\Psi_{f_{N}, \alpha}(I)$ is uniformly bounded (by a constant depending on the volume of $J$ ) when $I$ is a cube of sidelength $2^{k}$ for any integer $k$. By Lemma 2.1, $f_{N} \in Q_{\alpha}\left(\boldsymbol{R}^{n}\right)$, with norm

$$
\left\|f_{N}\right\|_{Q_{\alpha}\left(\boldsymbol{R}^{n}\right)} \leq C_{N, J} \sup _{t \in \boldsymbol{R}^{n}}\|F(t, \cdot)\|_{Q_{\alpha}^{(d)}\left(\boldsymbol{R}^{n}\right)} .
$$

3. Atomic decomposition for $Q_{\alpha}\left(\boldsymbol{R}^{n}\right)$. We follow the terminology of [FJ], Section 4, for the following:

Definition 3.1. We call a function $a_{I}$ a $(0, \infty)$-atom if there exists a cube $I \subset \boldsymbol{R}^{n}$ and integers $N_{1} \geq 1$ and $N_{2} \geq 0$, such that: 
(a) $\operatorname{supp} a_{I} \subset 3 I$;

(b) $\left|D^{\gamma} a_{I}(x)\right| \leq(\ell(I))^{-|\gamma|}$, for $D^{\gamma}=\left(\partial / \partial x_{1}\right)^{\gamma_{1}} \cdots\left(\partial / \partial x_{n}\right)^{\gamma_{n}}$ and $|\gamma| \leq N_{1}$;

(c) $\int_{\boldsymbol{R}^{n}} x^{\gamma} a_{I}(x) d x=0$, for $|\gamma| \leq N_{2}$.

Uchiyama proves (cf. [U], Lemmas 3.1-3.4) that $f \in \operatorname{BMO}\left(\boldsymbol{R}^{n}\right)$ if and only if $f$ can be written as

$$
f=\sum_{I \in \mathcal{D}} s_{I} a_{I}
$$

with $\left\{a_{I}\right\}_{I \in \mathcal{D}}$ a sequence of $(0, \infty)$-atoms and $\left\{s_{I}\right\}_{I \in \mathcal{D}}$ a sequence of coefficients such that the measure $\mu=\sum_{I}\left|s_{I}\right|^{2}|I| \delta_{\left(x_{I}, \ell(I)\right)}$ satisfies the Carleson condition

$$
\|\mu\|_{c}:=\sup _{I \in \mathcal{D}} \mu(S(I)) /|I|<\infty .
$$

Here $\delta_{(x, t)}$ is used to denote the unit mass at the point $(x, t)$ in the upper half-space $\boldsymbol{R}_{+}^{n+1}=$ $\left\{(x, t) ; x \in \boldsymbol{R}^{n}, t>0\right\}$, and $S(I)$ denotes the "Carleson box" above the cube $I$, i.e.

$$
S(I)=\left\{(y, t) \in \boldsymbol{R}_{+}^{n+1} ; y \in I, 0<t<\ell(I)\right\} .
$$

This condition on the measure is equivalent (cf. [FJ], Section 4) to the following condition on the coefficients (which we will also call the Carleson condition):

$$
\left\|\left\{s_{I}\right\}_{I \in \mathcal{D}}\right\|_{\mathcal{C}}:=\left(\sup _{I \in \mathcal{D}} \frac{1}{|I|} \sum_{J \subseteq I}\left|s_{J}\right|^{2}|J|\right)^{1 / 2}<\infty .
$$

In fact, in Uchiyama's result, condition (b) on the atoms is replaced by the Lipschitz condition $\left\|a_{I}\right\|_{\text {Lip1 }} \leq \ell(I)^{-1}$, where we use $\|f\|_{\text {Lip1 }}$ to denote the Lip1-norm of $f \in C\left(\boldsymbol{R}^{n}\right)$, namely

$$
\|f\|_{\text {Lip1 }}:=\sup _{x \neq y} \frac{|f(x)-f(y)|}{|x-y|},
$$

and in condition (c) Uchiyama has $N_{2}=0$. In $[\mathrm{FJ}]$ the result is stated with more smoothness and cancellation conditions on the atoms.

We will extend this result to $Q_{\alpha}\left(\boldsymbol{R}^{n}\right)$ for $\alpha \in(0,1)$. There is a similar characterization of $Q_{\alpha}\left(\boldsymbol{R}^{n}\right)$ in terms of wavelets, given in [EJPX], Section 6, whose proof is based on that of Meyer [M] for the case of BMO. However, for the atomic decomposition we cannot assume orthogonality and we need to compensate with quasi-orthogonality, as in Uchiyama [U]. The idea of the quasi-orthogonal decomposition goes back to Chang and Fefferman [CF] (see also [St], Section IV.4.5).

Definition 3.2. For $\alpha \in(-\infty, \infty), I \in \mathcal{D}$ and $\mathbf{s}=\left\{s_{K}\right\}_{K \in \mathcal{D}}$ (a sequence associated to all the dyadic cubes in $\boldsymbol{R}^{n}$ ), let

$$
U_{\mathrm{s}, \alpha}(I):=\sum_{K \subseteq I}\left(\frac{\ell(K)}{\ell(I)}\right)^{n-2 \alpha}\left|s_{K}\right|^{2} .
$$


We say that $\left\{s_{K}\right\}_{K \in \mathcal{D}}$ is $\mathcal{C}_{\alpha}$-sequence provided that

$$
\left\|\left\{s_{K}\right\}_{K \in \mathcal{D}}\right\|_{\mathcal{C}_{\alpha}}:=\left(\sup _{I \in \mathcal{D}} U_{\mathbf{s}, \alpha}(I)\right)^{1 / 2}<\infty .
$$

For $\alpha=0$, this is just the Carleson condition $\left\|\left\{s_{K}\right\}_{K \in \mathcal{D}}\right\|_{\mathcal{C}}<\infty$ above. Note that for $\alpha \geq 0$, a $\mathcal{C}_{\alpha}$-sequence is also a $\mathcal{C}_{0}$-sequence, since

$$
U_{\mathbf{s}, 0}(I) \leq U_{\mathbf{s}, \alpha}(I)
$$

Before we continue we need to recall a basic geometric fact about cubes in $\boldsymbol{R}^{n}$. If $I, J$ are cubes, $\ell(I)=a \ell(J)$, and $b I \cap c J \neq \emptyset$ for some positive numbers $a, b, c$, then

$$
I \subset(a+a b+c) J .
$$

In particular, if we restrict $I$ to be a dyadic cube, then for a fixed $J$ there are at most $(1+b+$ $c / a)^{n}$ such choices of $I$.

The next lemma extends Uchiyama's quasi-orthogonality result, Lemma 3.3 in [U] (see also [St], Lemma 4.5.1).

Lemma 3.3. Let $j \in N$ and suppose $\left\{s_{K}\right\}_{K \in \mathcal{D}}$ is a $\mathcal{C}_{\alpha}$-sequence. For integers $l<m$, consider a collection $\mathcal{F}$ of dyadic cubes such that $2^{l} \leq \ell(K) \leq 2^{m}$ for every element $K$ of $\mathcal{F}$. Define

$$
f(x)=\sum_{K \in \mathcal{F}} s_{K} a_{K}(x), \quad x \in \boldsymbol{R}^{n}
$$

where the functions $a_{K}$ satisfy:

(a') $\operatorname{supp} a_{K} \subseteq 2^{j} K$;

$\left(\mathrm{b}^{\prime}\right) \quad\left\|a_{K}\right\|_{\mathrm{Lip} 1} \leq 2^{-j}(\ell(K))^{-1}$;

(c') $\int_{\boldsymbol{R}^{n}} a_{K}(x) d x=0$.

If $\alpha \in[0,1)$, then there exists a constant $C$, independent of the choice of $l, m$ and $\mathcal{F}$, such that

(i)

$$
\|f\|_{L^{2}}^{2} \leq C 2^{2 j n} \sum_{K \in \mathcal{F}}\left|s_{K}\right|^{2} \ell(K)^{n}
$$

and

(ii)

$$
\iint \frac{\left|\sum_{\left\{K \in \mathcal{F} ; x, y \in 2^{j} K\right\}} s_{K}\left[a_{K}(x)-a_{K}(y)\right]\right|^{2}}{|x-y|^{n+2 \alpha}} d x d y \leq C 2^{2 j(n-\alpha)} \sum_{K \in \mathcal{F}}\left|s_{K}\right|^{2} \ell(K)^{n-2 \alpha} .
$$

Proof. Since $\alpha \geq 0,\left\{s_{K}\right\}$ is also $\mathcal{C}_{0}$-sequence and therefore part (i) is just Lemma 3.3 in [U]. We omit the proof since we will repeat the argument for the proof of part (ii). 
Suppose $J, K \in \mathcal{F}$ with $\ell(J) \leq \ell(K)$ and $2^{j} J \cap 2^{j} K \neq \emptyset$. Then

$$
\begin{aligned}
& \int_{2^{j} J} \int_{2^{j} K} \int_{2^{j} J \cap 2^{j} K} \frac{\left|a_{K}(x)-a_{K}(y)\right|\left|a_{J}(x)-a_{J}(y)\right|}{|x-y|^{n+2 \alpha}} d x d y \\
& \leq 2^{-2 j} \ell(K)^{-1} \ell(J)^{-1} \int_{2^{j} J} \int_{2^{j} J}|x-y|^{2-n-2 \alpha} d x d y \\
& \leq C 2^{j(n-2 \alpha)} \ell(K)^{-1} \ell(J)^{n+1-2 \alpha} .
\end{aligned}
$$

Now for each cube $K \in \mathcal{F}$ and integer $k \geq 0$, let

$$
\mathcal{G}_{k}(K)=\left\{J \in \mathcal{F} ; \ell(J)=2^{-k} \ell(K) \text { and } 2^{j} J \cap 2^{j} K \neq \emptyset\right\} .
$$

Take $\Omega$ to be a bounded set in $\boldsymbol{R}^{n}$, so that the number of $K \in \mathcal{F}$ for which $2^{j} K \cap \Omega \neq \emptyset$ is finite. Thus if we integrate over $\Omega \times \Omega$, we can, after expanding the square inside the integral, interchange the order of summation and integration (since the sum is finite) and use (3.2) to get

$$
\begin{aligned}
& \int_{\Omega} \int_{\Omega} \frac{\left|\sum_{\left\{K \in \mathcal{F}: x, y \in 2^{j} K\right\}} s_{K}\left[a_{K}(x)-a_{K}(y)\right]\right|^{2}}{|x-y|^{n+2 \alpha}} d x d y \\
& \leq \sum_{K \in \mathcal{F}}\left|s_{K}\right| \sum_{k=0}^{\infty} \sum_{J \in \mathcal{G}_{k}(K)}\left|s_{J}\right| \int_{2^{j} J \cap 2^{j} K} \int_{2^{j} J \cap 2^{j} K} \frac{\left|a_{K}(x)-a_{K}(y)\right|\left|a_{J}(x)-a_{J}(y)\right|}{|x-y|^{n+2 \alpha}} d x d y \\
& \leq C \sum_{k=0}^{\infty} \sum_{K \in \mathcal{F}}\left|s_{K}\right| \sum_{J \in \mathcal{G}_{k}(K)}\left|s_{J}\right| 2^{j(n-2 \alpha)} \ell(K)^{-1} \ell(J)^{n+1-2 \alpha} \\
& \leq C 2^{j(n-2 \alpha)} \sum_{k=0}^{\infty} 2^{-k(n+1-2 \alpha)}\left(\sum_{K \in \mathcal{F}}\left|s_{K}\right|^{2} \ell(K)^{n-2 \alpha}\right)^{1 / 2} \\
& \times\left(\sum_{K \in \mathcal{F}}\left(\sum_{\mathcal{G}_{k}(K)}\left|s_{J}\right|\right)^{2} \ell(K)^{n-2 \alpha}\right)^{1 / 2} \\
& \leq C 2^{j(n-2 \alpha)} \sum_{k=0}^{\infty} 2^{-k(n+1-2 \alpha)}\left(\sum_{K \in \mathcal{F}}\left|s_{K}\right|^{2} \ell(K)^{n-2 \alpha}\right)^{1 / 2} \\
& \times\left(\sum_{K \in \mathcal{F}} \sum_{\mathcal{G}_{k}(K)}\left|s_{J}\right|^{2} 2^{(j+k) n} \ell(K)^{n-2 \alpha}\right)^{1 / 2} \\
& \leq C 2^{j(n-2 \alpha)} \sum_{k=0}^{\infty} 2^{-k(n+1-2 \alpha)}\left(\sum_{K \in \mathcal{F}}\left|s_{K}\right|^{2} \ell(K)^{n-2 \alpha}\right)^{1 / 2} \\
& \times\left(\sum_{J \in \mathcal{F}}\left|s_{J}\right|^{2} 2^{2 j n+k(2 n-2 \alpha)} \ell(J)^{n-2 \alpha}\right)^{1 / 2} \\
& \leq C 2^{2 j(n-\alpha)} \sum_{k=0}^{\infty} 2^{-k(1-\alpha)} \sum_{K \in \mathcal{F}}\left|s_{K}\right|^{2} \ell(K)^{n-2 \alpha}
\end{aligned}
$$




$$
\leq C 2^{2 j(n-\alpha)} \sum_{K \in \mathcal{F}}\left|s_{K}\right|^{2} \ell(K)^{n-2 \alpha} \quad(\text { since } \alpha<1) .
$$

Note that we have used the the basic geometric fact above (see remark before the statement of the lemma) to bound the cardinality of $\mathcal{G}_{k}(K)$ by a constant multiple of $2^{(j+k) n}$ and the cardinality of $\left\{K: J \in \mathcal{G}_{k}(K)\right\}$ (for a fixed $J$ ) by a constant multiple of $2^{j n}$.

We have thus proved Part (ii) of the lemma for the double integral over any set $\Omega \times \Omega$, $\Omega \subset \boldsymbol{R}^{n}$ bounded, and therefore it holds for the integral over $\boldsymbol{R}^{n} \times \boldsymbol{R}^{n}$.

Continuing as in [U], we give an analogue of Uchiyama's Lemma 3.4 in the case of $Q_{\alpha}\left(\boldsymbol{R}^{n}\right)$.

LEMMA 3.4. Under the hypotheses of Lemma 3.3, if $0<\alpha<1$, we have

$$
\|f\|_{Q_{\alpha}\left(\boldsymbol{R}^{n}\right)} \leq C 2^{j n}\left\|\left\{s_{K}\right\}\right\|_{\mathcal{C}_{\alpha}},
$$

where the constant $C$ is independent of the choice of $\mathcal{F}$.

PROOF. Before we start, let us point out that one can remove all reference to $\mathcal{F}$ below by setting $s_{K}=0$ for $K \notin \mathcal{F}$.

The proof is similar in notation and outline to that of [EJPX], Theorem 6.2 (see also [M], p. 154). By Lemma 2.1, in order to estimate $\|f\|_{Q_{\alpha}\left(\boldsymbol{R}^{n}\right)}$, it suffices to bound $O_{f, \alpha}(I)$ for arbitrary cubes $I$ of dyadic sidelength. Fix such a cube $I$, and set

$$
\mathcal{A}(I)=\left\{K \in \mathcal{D} ; 2^{j} K \cap I \neq \emptyset\right\} .
$$

Note that condition ( $\left.\mathrm{a}^{\prime}\right)$ implies $a_{K}=0$ on $I$ if $K \notin \mathcal{A}(I)$, so that in the sum (3.1) defining $f$, when $x \in I$, the only cubes $K$ that appear are those with $K \in \mathcal{A}(I)$. Partition $\mathcal{A}(I)$ into

$$
\begin{aligned}
& \mathcal{A}_{1}=\mathcal{A}_{1}(I)=\left\{K \in \mathcal{A}(I) ; 2^{j} \ell(K) \leq \ell(I)\right\}, \\
& \mathcal{A}_{2}=\mathcal{A}_{2}(I)=\left\{K \in \mathcal{A}(I) ; \ell(I)<2^{j} \ell(K)\right\} .
\end{aligned}
$$

Then we have $f=f_{1}+f_{2}$ on $I$, where

$$
f_{i}=\sum_{K \in \mathcal{A}_{i}} s_{K} a_{K}, \quad i=1,2,
$$

and

$$
O_{f, \alpha}(I) \leq 2\left(O_{f_{1}, \alpha}(I)+O_{f_{2}, \alpha}(I)\right) .
$$

To take care of $f_{1}$, we again separate the sum into two parts:

$$
\begin{aligned}
O_{f_{1}, \alpha}(I)= & \ell(I)^{2 \alpha-n} \int_{I} \int_{I} \frac{\left|\sum_{K \in \mathcal{A}_{1}} s_{K}\left[a_{K}(x)-a_{K}(y)\right]\right|^{2}}{|x-y|^{n+2 \alpha}} d x d y \\
\leq & 2 \ell(I)^{2 \alpha-n} \int_{I} \int_{I} \frac{\left|\sum_{\left\{K \in \mathcal{A}_{1} ; x, y \in 2^{j+1} \sqrt{n} K\right\}} s_{K}\left[a_{K}(x)-a_{K}(y)\right]\right|^{2}}{|x-y|^{n+2 \alpha}} d x d y \\
& +4 \ell(I)^{2 \alpha-n} \int_{I} \int_{I} \frac{\left|\sum_{\left\{K \in \mathcal{A}_{1} ; x \in 2^{j} K, y \notin 2^{j+1} \sqrt{n} K\right\}} s_{K} a_{K}(x)\right|^{2}}{|x-y|^{n+2 \alpha}} d x d y
\end{aligned}
$$




$$
:=\ell(I)^{2 \alpha-n}(A+B) \text {. }
$$

Take an integer $m$ sufficiently large so that $2^{m} \geq 2 \sqrt{n}$, and apply Lemma 3.3, Part (ii), with $2^{-m} a_{K}$ instead of $a_{K}$ and $2^{j+m} K$ instead of $2^{j} K$. Then, since $\alpha \geq 0$,

$$
A \leq C_{m} 2^{2 j(n-\alpha)} \sum_{K \in \mathcal{A}_{1}}\left|s_{K}\right|^{2} \ell(K)^{n-2 \alpha} \leq C 2^{2 j n} \sum_{K \in \mathcal{A}_{1}}\left|s_{K}\right|^{2} \ell(K)^{n-2 \alpha}
$$

Noting that for $x \in 2^{j} K$ and $y \notin 2^{j+1} \sqrt{n} K,|x-y| \geq\left|x_{K}-y\right| / 2$, we can apply Part (i) of Lemma 3.3 to the functions $a_{K}(x)$ and coefficients

$$
\lambda_{K, y}=s_{K}\left|x_{K}-y\right|^{-n / 2-\alpha} \chi_{\left\{K ; y \notin 2^{j+1} \sqrt{n} K\right\}},
$$

for a fixed $y \in I$, to get

$$
\begin{aligned}
B & \leq C \int_{I} \int_{I}\left|\sum_{\left\{K \in \mathcal{A}_{1} ; x \in 2^{j} K, y \notin 2^{j+1} \sqrt{n} K\right\}} s_{K} a_{K}(x)\right| x_{K}-\left.\left.y\right|^{-n / 2-\alpha}\right|^{2} d x d y \\
& =C \int_{I}\left\|\sum_{K \in \mathcal{A}_{1}} \lambda_{K, y} a_{K}\right\|_{L^{2}(I)}^{2} d y \\
& \leq C 2^{2 j n} \int_{I} \sum_{K \in \mathcal{A}_{1}}\left|\lambda_{K, y}\right|^{2} \ell(K)^{n} d y \\
& \leq C 2^{2 j n} \sum_{K \in \mathcal{A}_{1}}\left|s_{K}\right|^{2} \ell(K)^{n} \int_{I \backslash 2^{j+1} \sqrt{n} K}\left|x_{K}-y\right|^{-n-2 \alpha} d y \\
& \leq C 2^{2 j n} \sum_{K \in \mathcal{A}_{1}}\left|s_{K}\right|^{2} \ell(K)^{n-2 \alpha} .
\end{aligned}
$$

Thus (3.4) and (3.5) give

$$
\begin{aligned}
O_{f_{1}, \alpha}(I) & \leq C 2^{2 j n} \ell(I)^{2 \alpha-n} \sum_{K \in \mathcal{A}_{1}}\left|s_{K}\right|^{2} \ell(K)^{n-2 \alpha} \\
& \leq C 2^{2 j n} \sum_{I^{\prime} \in \mathcal{E}(I)} \sum_{K \subseteq I^{\prime}}\left|s_{K}\right|^{2}\left(\frac{\ell(K)}{\ell\left(I^{\prime}\right)}\right)^{n-2 \alpha} \\
& \leq C 2^{2 j n} \sup _{I^{\prime} \in \mathcal{E}(I)} U_{\mathbf{s}, \alpha}\left(I^{\prime}\right),
\end{aligned}
$$

where we have used the notation $\mathcal{E}(I)$ to denote the collection of (at most $5^{n}$ ) dyadic cubes $I^{\prime}$ with $\ell\left(I^{\prime}\right)=\ell(I)$ for which $I^{\prime} \cap 3 I \neq \emptyset$. Note that for $K \in \mathcal{A}_{1}$, we have $\ell(K) \leq 2^{-j} \ell(I)$ and $2^{j} K \cap I \neq \emptyset$, which implies $K \subset 3 I$, hence $K \subseteq I^{\prime}$ for a unique $I^{\prime} \in \mathcal{E}(I)$. 
Now for $f_{2}$, by the Lipschitz condition $\left(\mathrm{b}^{\prime}\right)$ above, and using the fact that for every dyadic cube $K,\left|s_{K}\right|^{2} \leq U_{\mathbf{s}, \alpha}(K)$, we have

$$
\begin{aligned}
O_{f_{2}, \alpha}(I) & =C \ell(I)^{2 \alpha-n} \int_{I} \int_{I} \frac{\left|\sum_{K \in \mathcal{A}_{2}} s_{K}\left[a_{K}(x)-a_{K}(y)\right]\right|^{2}}{|x-y|^{n+2 \alpha}} d x d y \\
& \leq C \ell(I)^{2 \alpha-n} \int_{I} \int_{I}\left(\sum_{K \in \mathcal{A}_{2}}\left|s_{K}\right|\left(2^{j} \ell(K)\right)^{-1}\right)^{2}|x-y|^{2-n-2 \alpha} d x d y \\
& \leq C \ell(I)^{2 \alpha-n}\left(\sup _{K \in \mathcal{A}_{2}} U_{\mathbf{s}, \alpha}(K)\right)\left(\sum_{K \in \mathcal{A}_{2}}\left(2^{j} \ell(K)\right)^{-1}\right)^{2} \ell(I)^{2+n-2 \alpha} \\
& \left.\leq C\left(\sup _{K \in \mathcal{A}_{2}} U_{\mathbf{s}, \alpha}(K)\right)\left(\sum_{K \in \mathcal{A}_{2}} \frac{\ell(I)}{2^{j} \ell(K)}\right)^{2} 2^{-k}\right)^{2} \\
& \leq C\left(\sup _{K \in \mathcal{A}_{2}} U_{\mathbf{s}, \alpha}(K)\right)\left(\sum_{k=1}^{\infty} \sum_{K \in \mathcal{A}_{2}, 2^{j} \ell(K)=2^{k} \ell(I)} \operatorname{sip}^{2 j n}\left(\sup _{K \in \mathcal{A}_{2}} U_{\mathbf{s}, \alpha}(K)\right) .\right.
\end{aligned}
$$

Here we have again used the geometric fact that for each $k \in N$, there are at most $2^{(j+1) n}$ choices of $K \in \mathcal{A}_{2}$ with $2^{j} \ell(K)=2^{k} \ell(I)$.

Therefore, by estimates (3.3), (3.6) and (3.7), we conclude

$$
O_{f, \alpha}(I) \leq C 2^{2 n j}\left(\sup _{I^{\prime} \in \mathcal{E}(I)} U_{\mathbf{s}, \alpha}\left(I^{\prime}\right)+\sup _{K \in \mathcal{A}_{2}(I)} U_{\mathbf{s}, \alpha}(K)\right) \leq C 2^{2 n j}\left\|\left\{s_{K}\right\}\right\|_{\mathcal{C}_{\alpha}}^{2}
$$

for every cube $I$ of dyadic sidelength. By Lemma 2.1, we have $f \in Q_{\alpha}\left(\boldsymbol{R}^{n}\right)$ with norm $\|f\|_{Q_{\alpha}\left(\boldsymbol{R}^{n}\right)} \leq C 2^{j n}\left\|\left\{s_{K}\right\}\right\|_{\mathcal{C}_{\alpha}}$. Note that nowhere in the proof did we use the number of elements of $\mathcal{F}$.

Before getting to the main theorem of this section, we need to review some results from [DX]. We first state a lemma which is a combination of Lemma 1.1 in [FJW] and Lemma 3.2 in [DX]. Here and below we will denote the Schwartz class of rapidly decreasing smooth functions on $\boldsymbol{R}^{n}$ by $\mathcal{S}$, and its dual, the space of tempered distributions, by $\mathcal{S}^{\prime}$. For a function $\phi \in \mathcal{S}\left(\boldsymbol{R}^{n}\right), \hat{\phi}$ will denote the Fourier transform of $\phi$.

LEMmA 3.5. Fix $N \in N$. Then there exists a function $\phi: \boldsymbol{R}^{n} \rightarrow \boldsymbol{R}$ such that

(1) $\operatorname{supp} \phi \subset\left\{x \in \boldsymbol{R}^{n} ;|x| \leq 1\right\}$;

(2) $\phi$ is radial;

(3) $\phi \in C^{\infty}\left(\boldsymbol{R}^{n}\right)$;

(4) $\int_{\boldsymbol{R}^{n}} x^{\gamma} \phi(x) d x=0$ if $|\gamma| \leq N, \gamma \in(\boldsymbol{N} \cup\{0\})^{n}, x^{\gamma}=x_{1}^{\gamma_{1}} x_{2}^{\gamma_{2}} \cdots x_{n}^{\gamma_{n}},|\gamma|=$ $\gamma_{1}+\gamma_{2}+\cdots+\gamma_{n}$

(5) $\int_{0}^{\infty}(\hat{\phi}(t \xi))^{2} t^{-1} d t=1$ if $\xi \in \boldsymbol{R}^{n} \backslash\{0\}$. 
Moreover, if $\alpha \in(0,1), f \in L_{\mathrm{loc}}^{2}\left(\boldsymbol{R}^{n}\right)$, and $d \mu_{f, \phi, \alpha}(x, t)=\left|\left(f * \phi_{t}\right)(x)\right|^{2} t^{-1-2 \alpha} d t d x$, then there is a constant $C$, independent of the choice of $f$, such that for any cubes $I$ and $J$ in $\boldsymbol{R}^{n}$, with center $x_{I}=x_{J}$ and with $\ell(J) \geq 3 \ell(I)$,

$$
\mu_{f, \phi, \alpha}(S(I)) \leq C \ell(J)^{n-2 \alpha} O_{f, \alpha}(J) .
$$

Here again $S(I)$ is the "Carleson box" over $I$.

Next, we need to recall the duality result in [DX], identifying $Q_{\alpha}\left(\boldsymbol{R}^{n}\right)$ with the dual of the "Hardy-Hausdorff space" $H H_{-\alpha}^{1}\left(\boldsymbol{R}^{n}\right)$. This space can be characterized by the following atomic decomposition (Theorem 6.3 in $[\mathrm{DX}]$ ):

THEOREM 3.6(DX). Let $0<\alpha<\min \{1, n / 2\}$. Define an $H H_{-\alpha}^{1}$-atom a to be a tempered distribution supported in a cube I and satisfying:

(i)

$$
|\langle a, \psi\rangle| \leq\left(O_{\psi, \alpha}(I)\right)^{1 / 2}
$$

for all $\psi \in \mathcal{S}$; and

(ii)

$$
\langle a, \psi\rangle=0
$$

for any $\psi \in \mathcal{S}$ which coincides in a neighborhood of I with a polynomial of degree $\leq n / 2+1$.

Then a tempered distribution $f$ on $\boldsymbol{R}^{n}$ belongs to $H_{H_{\alpha}}^{1}$ if and only if there is a sequence of $H_{-\alpha}^{1}$-atoms $\left\{a_{j}\right\}$, and an $l^{1}$ sequence $\left\{\lambda_{j}\right\}$, such that $f=\sum_{j} \lambda_{j} a_{j}$ in the sense of distributions. Moreover,

$$
\|f\|_{H H_{-\alpha}^{1}\left(\boldsymbol{R}^{n}\right)} \approx \inf \left\{\sum_{j}\left|\lambda_{j}\right| ; \quad f=\sum_{j} \lambda_{j} a_{j}\right\} .
$$

As explained in [DX] (see Remark 2 after Lemma 6.2), an $H H_{-\alpha}^{1}$-atom $a$ is actually a distribution in the homogeneous Sobolev space $\dot{L}_{-\alpha}^{2}\left(\boldsymbol{R}^{n}\right)$, and can thus be paired with a function $\psi$ in the dual homogeneous Sobolev space $\dot{L}_{\alpha}^{2}\left(\boldsymbol{R}^{n}\right)$, namely a function satisfying

$$
\int_{\boldsymbol{R}^{n}} \int_{\boldsymbol{R}^{n}} \frac{|\psi(x)-\psi(y)|^{2}}{|x-y|^{n+2 \alpha}} d x d y<\infty .
$$

In particular, we can take for $\psi$ any Lipschitz function with compact support, such as a $(0, \infty)$-atom. Moreover, approximating $\psi$ in $\dot{L}_{\alpha}^{2}\left(\boldsymbol{R}^{n}\right)$ by functions in $\mathcal{S}$, we see that condition (i) in Theorem 3.6 extends to the pairing of $a$ with such $\psi$.

We are now in a position to prove the major result of this section.

THEOREM 3.7. Let $0<\alpha<\min \{1, n / 2\}$. If $\left\{a_{I}\right\}_{I \in \mathcal{D}}$ is a sequence of $(0, \infty)$-atoms, and $\left\{s_{I}\right\}_{I \in \mathcal{D}}$ a $\mathcal{C}_{\alpha}$-sequence, then there exists a function $f \in Q_{\alpha}\left(\boldsymbol{R}^{n}\right)$ so that

$$
f=\sum_{I \in \mathcal{D}} s_{I} a_{I}=\lim _{k \rightarrow-\infty, m \rightarrow \infty} \sum_{I \in \mathcal{D}, 2^{k} \leq \ell(I) \leq 2^{m}} s_{I} a_{I},
$$

where the convergence is in $\mathcal{S}^{\prime}\left(\boldsymbol{R}^{n}\right)$ modulo constants and in the weak-* topology in $Q_{\alpha}\left(\boldsymbol{R}^{n}\right)$ (viewed as the dual of $H_{-\alpha}^{1}\left(\boldsymbol{R}^{n}\right)$ ). 
Conversely, if $f \in Q_{\alpha}\left(\boldsymbol{R}^{n}\right)$ then there is a sequence $\left\{a_{I}\right\}_{I \in \mathcal{D}}$ of $(0, \infty)$-atoms, and a $\mathcal{C}_{\alpha}$-sequence $\left\{s_{I}\right\}_{I \in \mathcal{D}}$ such that (3.9) holds. Moreover,

$$
\|f\|_{Q_{\alpha}\left(\boldsymbol{R}^{n}\right)} \approx\left\|\left\{s_{I}\right\}\right\|_{\mathcal{C}_{\alpha}} .
$$

Proof. Let $\left\{s_{I}\right\}_{I \in \mathcal{D}}$ be a $\mathcal{C}_{\alpha}$-sequence, and $\left\{a_{I}\right\}_{I \in \mathcal{D}}$ a sequence of $(0, \infty)$-atoms. Note that $a_{I} / 4$ satisfy conditions $\left(\mathrm{a}^{\prime}\right)-\left(\mathrm{c}^{\prime}\right)$ of Lemma 3.3 with $j=2$. Therefore if we denote by $\mathcal{F}_{k, m}$ the collection of cubes $K \in \mathcal{D}$ with $2^{k}<\ell(K) \leq 2^{m}$, and set

$$
f_{k, m}=\sum_{I \in \mathcal{F}_{k, m}} s_{I} a_{I}, \quad \text { for } k, m \in Z, \quad k \leq m,
$$

then by Lemma 3.4,

$$
\left\|f_{k, m}\right\|_{Q_{\alpha}\left(\boldsymbol{R}^{n}\right)} \leq C\left\|\left\{s_{I}\right\}\right\|_{\mathcal{C}_{\alpha}},
$$

with a constant independent of $k$ and $m$. From an analogous result for BMO (see [FJ], Theorem 4.1, or [St], Proposition IV.4.5), we know that as $k \rightarrow-\infty, m \rightarrow \infty, f_{k, m}$ converge in $\mathcal{S}^{\prime} / \boldsymbol{C}$ and weak-* in BMO (as the dual of $H^{1}$ ) to a function $f \in$ BMO. We want to show that $f \in Q_{\alpha}\left(\boldsymbol{R}^{n}\right)$ and in fact the convergence is weak-* in $Q_{\alpha}\left(\boldsymbol{R}^{n}\right)$, viewed as the dual of $H H_{-\alpha}^{1}\left(\boldsymbol{R}^{n}\right)$.

First, for $k \leq 0 \leq m$, write

$$
f_{k, m}=f_{k, 0}+f_{0, m} .
$$

Suppose $g$ is an $H H_{-\alpha}^{1}$-atom, supported in a cube $I$. Let $\tilde{I}$ be the smallest concentric cube containing $I$ with dyadic sidelength, say $\ell(\tilde{I})=2^{r}$.

Then for $0<m<p$, we have, by condition (i) in Theorem 3.6,

$$
\left|\left\langle f_{0, m}-f_{0, p}, g\right\rangle\right|^{2}=\left|\left\langle f_{m, p}, g\right\rangle\right|^{2} \leq C O_{f_{m, p}, \alpha}(I) \leq C O_{f_{m, p}, \alpha}(\tilde{I}) .
$$

If $m$ is sufficiently large $(m \geq r-2)$, we have $\ell(K)>\ell(\tilde{I}) / 4$ for all $K \in \mathcal{F}_{m, p}$, and hence we can repeat the calculations leading up to estimate (3.7) in the proof of Lemma 3.4 (with $j=2, \mathcal{F}=\mathcal{F}_{m, p}$ and $\left.\mathcal{A}_{2}=\{K \in \mathcal{D} ; \ell(K)>\ell(\tilde{I}) / 4,4 K \cap \tilde{I} \neq \emptyset\}\right)$ to get

$$
\begin{aligned}
O_{f_{m, p}, \alpha}(\tilde{I}) & \leq C\left(\sup _{K \in \mathcal{A}_{2}} U_{\mathbf{s}, \alpha}(K)\right)\left(\sum_{k=1}^{\infty} \sum_{K \in \mathcal{F}_{m, p}, \ell(K)=2^{k} \ell(\tilde{I}) / 4} 2^{-k}\right)^{2} \\
& \leq C 2^{4 n}\left\|\left\{s_{K}\right\}\right\|_{\mathcal{C}_{\alpha}}^{2}\left(\sum_{k>m+2-r} 2^{-k}\right)^{2} .
\end{aligned}
$$

This shows $\left\langle f_{0, m}-f_{0, p}, g\right\rangle \rightarrow 0$ as $m, p \rightarrow \infty$.

To show $\left\langle f_{q, 0}-f_{k, 0}, g\right\rangle \rightarrow 0$ as $q, k \rightarrow-\infty$ we can use estimate (3.6), since for $K$ in $\mathcal{F}_{q, k}, q<k \leq r-2$, we have $\ell(K) \leq \ell(\tilde{I}) / 4$. This means that

$$
\left|\left\langle f_{q, 0}-f_{k, 0}, g\right\rangle\right|^{2} \leq C O_{f_{q, k}, \alpha}(\tilde{I})
$$




$$
\leq C 2^{4 n} \sum_{I^{\prime} \in \mathcal{E}(\tilde{I})} \sum_{K \in \mathcal{F}_{q, k}, K \subseteq I^{\prime}}\left|s_{K}\right|^{2}\left(\frac{\ell(K)}{\ell\left(I^{\prime}\right)}\right)^{n-2 \alpha}
$$

where

$$
\mathcal{E}(\tilde{I})=\left\{I^{\prime} \in \mathcal{D} ; \ell\left(I^{\prime}\right)=\ell(\tilde{I}), I^{\prime} \cap 3 \tilde{I} \neq \emptyset\right\} .
$$

Now $\mathcal{E}(\tilde{I})$ has at most $5^{n}$ elements, and for each of the cubes $I^{\prime} \in \mathcal{E}(\tilde{I})$, the finiteness of $U_{\mathbf{s}, \alpha}\left(I^{\prime}\right)$ implies that

$$
\sum_{K \in \mathcal{F}_{q, k}, K \subseteq I^{\prime}}\left(\frac{\ell(K)}{\ell\left(I^{\prime}\right)}\right)^{n-2 \alpha}\left|s_{K}\right|^{2} \rightarrow 0 \quad \text { as } q, k \rightarrow-\infty,
$$

giving $\left\langle f_{q, 0}-f_{k, 0}, g\right\rangle \rightarrow 0$ as $q, k \rightarrow-\infty$.

We have thus shown that $\lim _{k \rightarrow-\infty, m \rightarrow \infty}\left\langle f_{k, m}, g\right\rangle$ exists for any $g$ which is an $H H_{-\alpha^{-}}^{1}$ atom, or a finite linear combination of such atoms. Since the finite linear combinations of $H H_{-\alpha}^{1}$-atoms form a dense subset of the predual $H H_{-\alpha}^{1}\left(\boldsymbol{R}^{n}\right)$, and by (3.10) the sequence $\left\{f_{k, m}\right\}$ is uniformly bounded in $Q_{\alpha}\left(\boldsymbol{R}^{n}\right)$, we conclude that $f_{k, m}$ converge weak-* in $Q_{\alpha}\left(\boldsymbol{R}^{n}\right)$ to some function in $Q_{\alpha}\left(\boldsymbol{R}^{n}\right)$. This must be the same as the function $f$ (the weak-* limit in BMO), since $H^{1}\left(\boldsymbol{R}^{n}\right) \subset H H_{-\alpha}^{1}\left(\boldsymbol{R}^{n}\right)$ (see [DX]). Thus $f \in Q_{\alpha}\left(\boldsymbol{R}^{n}\right)$ with

$$
\|f\|_{Q_{\alpha}\left(\boldsymbol{R}^{n}\right)} \leq C\left\|\left\{s_{K}\right\}\right\|_{\mathcal{C}_{\alpha}} .
$$

Now let $f \in Q_{\alpha}\left(\boldsymbol{R}^{n}\right)$. For the atomic decomposition, we will follow the construction in the proof of Lemma 3.1 in [U], which in turn is based on [CF]. Let $\phi$ be as in Lemma 3.5. Then we can use Calderón's reproducing formula to obtain:

$$
\begin{aligned}
f(x) & =\int_{0}^{\infty}\left(\phi_{t} * \phi_{t} * f\right)(x) \frac{d t}{t} \\
& =\sum_{I \in \mathcal{D}} \int_{T(I)} \phi_{t}(x-y)\left(\phi_{t} * f\right)(y) \frac{d y d t}{t} \\
& =\sum_{I \in \mathcal{D}} b_{I}(x),
\end{aligned}
$$

where $T(I)$ is the upper half of the "Carleson box", namely

$$
T(I)=\left\{(y, t) \in \boldsymbol{R}_{+}^{n+1} ; y \in I, \ell(I) / 2 \leq t<\ell(I)\right\},
$$

and the convergence is in the sense of distributions modulo constants (i.e. in $\mathcal{S}^{\prime}\left(\boldsymbol{R}^{n}\right) / \boldsymbol{C}$ see, for example, [FJW], Appendix), or alternatively, in the weak-* sense in BMO (see [St], Section IV.4.5.3).

From the support and cancellation conditions on $\phi$, we can conclude that $b_{I}$ is supported in $3 I$ and $\int_{\boldsymbol{R}^{n}} x^{\gamma} b_{I}(x) d x=0$ for $|\gamma| \leq N=N_{2}$. Moreover, as in the proof of Lemma 3.1 in [U] (for higher derivatives see also [St], Section IV.4.5.3), we can differentiate inside the integral to obtain

$$
\left|D_{x}^{\gamma} b_{I}(x)\right|=\left|\int_{T(I)}\left(D_{x}^{\gamma} \phi_{t}(x-y)\right)\left(\phi_{t} * f\right)(y) \frac{d y d t}{t}\right|
$$




$$
\begin{aligned}
& \leq\left\|D^{\gamma} \phi\right\|_{L^{2}}\left(\int_{\ell(I) / 2}^{\ell(I)} t^{-n-2|\gamma|-1} d t\right)^{1 / 2}\left(\int_{T(I)}\left|\left(\phi_{t} * f\right)(y)\right|^{2} \frac{d y d t}{t}\right)^{1 / 2} \\
& \leq C_{\phi, \gamma} \ell(I)^{-n / 2-|\gamma|}\left(\int_{T(I)}\left|\left(\phi_{t} * f\right)(y)\right|^{2} \frac{d y d t}{t}\right)^{1 / 2} .
\end{aligned}
$$

Thus if we let $a_{I}=b_{I} / s_{I}$, where

$$
s_{I}=C_{N_{2}}|I|^{-1 / 2}\left(\int_{T(I)}\left|\left(\phi_{t} * f\right)(y)\right|^{2} t^{-1} d t d y\right)^{1 / 2}
$$

and $C_{N_{2}}$ is so chosen to be larger than $C_{\phi, \gamma}$ for all $|\gamma| \leq N_{2}$, then $a_{I}$ is a $(0, \infty)$-atom and $f=\sum_{I \in \mathcal{D}} s_{I} a_{I}$ in $\mathcal{S}^{\prime}\left(\boldsymbol{R}^{n}\right) / \boldsymbol{C}$.

In order to verify that $\left\{s_{I}\right\}_{I \in \mathcal{D}}$ is a $\mathcal{C}_{\alpha}$-sequence, we apply Lemma 3.5 to obtain that for any $I \in \mathcal{D}$,

$$
\begin{aligned}
U_{\mathbf{s}, \alpha}(I) & =C(\ell(I))^{2 \alpha-n} \sum_{J \subseteq I} \ell(J)^{n-2 \alpha}|J|^{-1} \int_{T(J)}\left|\left(\phi_{t} * f\right)(y)\right|^{2} t^{-1} d t d y \\
& \leq C(\ell(I))^{2 \alpha-n} \sum_{J \subseteq I} \int_{T(J)}\left|\left(\phi_{t} * f\right)(y)\right|^{2} t^{-1-2 \alpha} d t d y \\
& =C(\ell(I))^{2 \alpha-n} \int_{S(I)}\left|\left(\phi_{t} * f\right)(y)\right|^{2} t^{-1-2 \alpha} d t d y \\
& \leq C O_{f, \alpha}(3 I)
\end{aligned}
$$

so taking the supremum over $I$,

$$
\left\|\left\{s_{I}\right\}\right\|_{\mathcal{C}_{\alpha}} \leq C\|f\|_{Q_{\alpha}\left(\boldsymbol{R}^{n}\right)}
$$

Finally, note that by the first part of the theorem, $\sum s_{I} a_{I}$ converge in the weak- $*$ sense to a limit in $Q_{\alpha}\left(\boldsymbol{R}^{n}\right)$, and since we already have the weak-* convergence in BMO to $f$, this limit must be $f$. By (3.11) and (3.12), $\|f\|_{Q_{\alpha}\left(\boldsymbol{R}^{n}\right)} \approx\left\|\left\{s_{I}\right\}\right\|_{\mathcal{C}_{\alpha}}$, as desired.

This completes the proof of the theorem.

Note that in proving the weak-* convergence in $Q_{\alpha}\left(\boldsymbol{R}^{n}\right)$ of the sum of atoms $\sum s_{I} a_{I}$, we did not use the full force of Lemma 3.4, but rather only the case $j=2$. In fact, using Lemma 3.4 and the following lemma of Uchiyama (Lemma 3.5 in [U]), we can prove a stronger result.

LEMMA 3.8 (Uchiyama). Let $I \subset \boldsymbol{R}^{n}$ be a cube with center $x_{I}$, and suppose $b \in$ $C^{1}\left(\boldsymbol{R}^{n}\right)$ satisfies

(i) $\int_{\boldsymbol{R}^{n}} b(x) d x=0$;

(ii) $|b(x)| \leq(\ell(I))^{n+1} /\left(\ell(I)+\left|x-x_{I}\right|\right)^{n+1}$;

(iii) $\left|\partial_{x_{i}} b(x)\right| \leq(\ell(I))^{n+1} /\left(\ell(I)+\left|x-x_{I}\right|\right)^{n+2}$ for $i=1, \ldots, n$. 
Then there exists a sequence $\left\{a_{j}\right\}_{j=0}^{\infty}$ of functions in $C^{1}\left(\boldsymbol{R}^{n}\right)$ such that

$$
b(x)=\sum_{j=0}^{\infty} 2^{-j(n+1)} a_{j}(x),
$$

and for each $j$ the function $a_{j}$ satisfies conditions $\left(\mathrm{a}^{\prime}\right)-\left(\mathrm{c}^{\prime}\right)$ of Lemma 3.3 (with respect to the cube I).

We will call a function $b \in C^{1}\left(\boldsymbol{R}^{n}\right)$ satisfying conditions (i) - (iii) of Lemma 3.8 a $(0, \infty)$-molecule (see $[\mathrm{FJ}]$, Section 3 ). Note that every $(0, \infty)$-atom is also a molecule (up to a constant). Conversely, the lemma may be thought of as the decomposition of a molecule into atoms. We now state the extension of Theorem 3.7, namely the weak-* convergence in $Q_{\alpha}\left(\boldsymbol{R}^{n}\right)$ of a sum of molecules. An analogous result for BMO is Theorem 4.1(b) in [FJ].

THEOREM 3.9. Let $0<\alpha<\min \{1, n / 2\}$. If $\left\{b_{I}\right\}_{I \in \mathcal{D}}$ is a sequence of $(0, \infty)$ molecules, and $\left\{s_{I}\right\}_{I \in \mathcal{D}}$ a $\mathcal{C}_{\alpha}$-sequence, then there exists a function $f \in Q_{\alpha}\left(\boldsymbol{R}^{n}\right)$ so that

$$
f=\sum_{I \in \mathcal{D}} s_{I} b_{I}=\lim _{k \rightarrow-\infty, m \rightarrow \infty} \sum_{I \in \mathcal{D}, 2^{k} \leq \ell(I) \leq 2^{m}} s_{I} b_{I},
$$

where the convergence is in $\mathcal{S}^{\prime}\left(\boldsymbol{R}^{n}\right)$ modulo constants and in the weak-* topology in $Q_{\alpha}\left(\boldsymbol{R}^{n}\right)$ (viewed as the dual of $\mathrm{HH}_{-\alpha}^{1}\left(\boldsymbol{R}^{n}\right)$ ).

Proof. Let $\left\{s_{I}\right\}_{I \in \mathcal{D}}$ be a $\mathcal{C}_{\alpha}$-sequence, and $\left\{a_{I}\right\}_{I \in \mathcal{D}}$ a sequence of $(0, \infty)$-molecules. By Lemma 3.8,

$$
b_{I}(x)=\sum_{j=0}^{\infty} 2^{-j(n+1)} a_{I, j}(x),
$$

where each $a_{I, j}$ possesses properties $\left(\mathrm{a}^{\prime}\right)-\left(\mathrm{c}^{\prime}\right)$ of Lemma 3.3 with respect to $I$ and $j$. Following the proof of Theorem 3.7, we denote by $\mathcal{F}_{k, m}$ the collection of cubes $K \in \mathcal{D}$ with $2^{k}<\ell(K) \leq 2^{m}$, and set

$$
f_{k, m, l}(x)=\sum_{I \in \mathcal{F}_{k, m}} s_{I} \sum_{j=0}^{l} 2^{-j(n+1)} a_{I, j}(x), \quad \text { for } k, m, l \in \boldsymbol{Z}, \quad k \leq m, l \geq 0 .
$$

With help of Lemma 3.4 we obtain that

$$
\begin{aligned}
\left\|f_{k, m, l}\right\|_{Q_{\alpha}\left(\boldsymbol{R}^{n}\right)} & \leq\left\|\sum_{I \in \mathcal{F}_{k, m}} s_{I} \sum_{j=0}^{l} 2^{-j(n+1)} a_{I, j}\right\|_{Q_{\alpha}\left(\boldsymbol{R}^{n}\right)} \\
& \leq \sum_{j=0}^{l} 2^{-j(n+1)}\left\|\sum_{I \in \mathcal{F}_{k, m}} s_{I} a_{I, j}\right\|_{Q_{\alpha}\left(\boldsymbol{R}^{n}\right)} \\
& \leq C \sum_{j=0}^{\infty} 2^{-j(n+1)} 2^{j n}\left\|\left\{s_{I}\right\}\right\|_{\mathcal{C}_{\alpha}} \\
& \leq C\left\|\left\{s_{I}\right\}\right\|_{\mathcal{C}_{\alpha}}
\end{aligned}
$$


with $C$ independent of $k, m$ and $l$. Similarly,

$$
\left\|f_{k, m, l_{1}}-f_{k, m, l_{2}}\right\|_{Q_{\alpha}\left(\boldsymbol{R}^{n}\right)} \leq C \sum_{j=l_{1}}^{l_{2}} 2^{-j(n+1)} 2^{j n}\left\|\left\{s_{I}\right\}\right\|_{\mathcal{C}_{\alpha}} \rightarrow 0 \quad \text { as } l_{1}, l_{2} \rightarrow \infty .
$$

Thus $\lim _{l \rightarrow \infty} f_{k, m, l}$ exists in $Q_{\alpha}\left(\boldsymbol{R}^{n}\right)$, and since

$$
f_{k, m}(x)=\lim _{l \rightarrow \infty} f_{k, m, l}(x)=\sum_{I \in \mathcal{F}_{k, m}} s_{I} \sum_{j=0}^{\infty} 2^{-j(n+1)} a_{I, j}(x)
$$

converges pointwise, an application of Fatou's lemma gives $f_{k, m}=\lim _{l \rightarrow \infty} f_{k, m, l}$ in $Q_{\alpha}\left(\boldsymbol{R}^{n}\right)$. Moreover, by (3.13),

$$
\sup _{k, m}\left\|f_{k, m}\right\|_{Q_{\alpha}\left(\boldsymbol{R}^{n}\right)} \leq C\left\|\left\{s_{I}\right\}\right\|_{\mathcal{C}_{\alpha}} .
$$

As in the proof of Theorem 3.7, in order to show that $f_{k, m}$ converges weak-* in $Q_{\alpha}\left(\boldsymbol{R}^{n}\right)$, it remains to show that $\lim _{k \rightarrow-\infty, m \rightarrow \infty}\left\langle f_{k, m}, g\right\rangle$ exists for $g$ in a dense subset of the predual $H H_{-\alpha}^{1}\left(\boldsymbol{R}^{n}\right)$.

Again write $f_{k, m}=f_{k, 0}+f_{0, m}$ for $k \leq 0 \leq m$, take $g$ to be an $H H_{-\alpha}^{1}$-atom supported in a cube $I$, and let $\tilde{I}$ be the smallest concentric cube containing $I$ with dyadic sidelength. Then by condition (i) in Theorem 3.6, Fatou's lemma, and Minkowski's inequality, we have, for $0<m<p$,

$$
\begin{aligned}
\left|\left\langle f_{0, m}-f_{0, p}, g\right\rangle\right| & \leq C\left(O_{f_{0, m}-f_{0, p}, \alpha}(I)\right)^{1 / 2} \\
& \leq C\left(O_{f_{0, m}-f_{0, p}, \alpha}(\tilde{I})\right)^{1 / 2} \\
& \leq C \liminf _{l \rightarrow \infty}\left(O_{f_{0, m, l}-f_{0, p, l}, \alpha}(\tilde{I})\right)^{1 / 2} \\
& \leq C \liminf _{l \rightarrow \infty} \sum_{j=0}^{l} 2^{-j(n+1)}\left(O_{h_{j}, \alpha}(\tilde{I})\right)^{1 / 2} \\
& =C \sum_{j=0}^{\infty} 2^{-j(n+1)}\left(O_{h_{j}, \alpha}(\tilde{I})\right)^{1 / 2}
\end{aligned}
$$

where

$$
h_{j}=\sum_{K \in \mathcal{F}_{m, p}} s_{K} a_{K, j} .
$$

Imitating (3.7) in the proof of Lemma 3.4 (with $\mathcal{F}=\mathcal{F}_{m, p}, 2^{m} \geq 2^{-j} \ell(\tilde{I})$ ), we have

$$
\begin{aligned}
O_{h_{j}, \alpha}(\tilde{I}) & \leq C\left(\sup _{K \in \mathcal{A}_{2}(\tilde{I})} U_{\mathbf{s}, \alpha}(K)\right)\left(\sum_{k=1}^{\infty} \sum_{K \in \mathcal{F}_{m, p} \cap \mathcal{A}_{2}(\tilde{I}), 2 j \ell(K)=2^{k} \ell(\tilde{I})} 2^{-k}\right)^{2} \\
& \leq C 2^{2 j n}\left\|\left\{s_{K}\right\}\right\|_{\mathcal{C}_{\alpha}}^{2}\left(\sum_{k>m+j-\log _{2} \ell(\tilde{I})} 2^{-k}\right)^{2} \\
& =C 2^{-2 m+2 j(n-1)}\left\|\left\{s_{K}\right\}\right\|_{\mathcal{C}_{\alpha}}^{2} \ell(\tilde{I})^{2}
\end{aligned}
$$


Thus

as $m, p \rightarrow \infty$.

$$
\left|\left\langle f_{0, m}-f_{0, p}, g\right\rangle\right| \leq C 2^{-m}\left\|\left\{s_{K}\right\}\right\|_{\mathcal{C}_{\alpha}} \ell(\tilde{I}) \sum_{j=0}^{\infty} 2^{-2 j} \rightarrow 0
$$

For the case of $\left\langle f_{q, 0}-f_{k, 0}, g\right\rangle$ as $k, q \rightarrow-\infty$, we repeat (3.14) with $h_{j}$ now standing for $\sum_{K \in \mathcal{F}_{q, k}} s_{K} a_{K, j}$. Assuming $q<k \leq \log _{2} \ell(\tilde{I})-j$ so that $K \in \mathcal{F}_{q, k}$ implies $2^{j} \ell(K) \leq \ell(\tilde{I})$, another application of (3.6) in the proof of Lemma 3.4 gives

$$
O_{h_{j}, \alpha}(\tilde{I}) \leq C 2^{2 j n} \sum_{I^{\prime} \in \mathcal{E}(\tilde{I})} \sum_{K \in \mathcal{F}_{q, k}, K \subseteq I^{\prime}}\left|s_{K}\right|^{2}\left(\frac{\ell(K)}{\ell\left(I^{\prime}\right)}\right)^{n-2 \alpha},
$$

where we recall that

$$
\mathcal{E}(\tilde{I})=\left\{I^{\prime} \in \mathcal{D} ; \ell\left(I^{\prime}\right)=\ell(\tilde{I}), I^{\prime} \cap 3 \tilde{I} \neq \emptyset\right\} .
$$

Noting again that $\mathcal{E}(\tilde{I})$ has at most $5^{n}$ elements, and for each of the cubes $I^{\prime} \in \mathcal{E}^{\prime}(\tilde{I})$, $\sum_{K \in \mathcal{F}_{q, k}, K \subseteq I^{\prime}}\left(\ell(K) / \ell\left(I^{\prime}\right)\right)^{n-2 \alpha}\left|s_{K}\right|^{2} \rightarrow 0 \quad$ as $q, k \rightarrow-\infty$ by the convergence of the series defining $U_{\mathbf{s}, \alpha}\left(I^{\prime}\right)$, we have that for each $j \geq 0,2^{-j n} O_{h_{j}, \alpha}(\tilde{I})^{1 / 2} \rightarrow 0$ as $q, k \rightarrow-\infty$. Moreover, $2^{-j n} O_{h_{j}, \alpha}(\tilde{I})^{1 / 2}$ are bounded by a constant multiple of $\sup _{I \in \mathcal{D}} U_{\mathbf{s}, \alpha}(I)^{1 / 2}=$ $\left\|\left\{s_{K}\right\}_{K \in \mathcal{D}}\right\|_{\mathcal{C}_{\alpha}}$. This, together with (3.14), implies that $\left\langle f_{q, 0}-f_{k, 0}, g\right\rangle \rightarrow 0$ as $q, k \rightarrow-\infty$.

The proof of weak-* convergence in $Q_{\alpha}\left(\boldsymbol{R}^{n}\right)$ is now complete. The convergence in $\mathcal{S}^{\prime} / \boldsymbol{C}$ is proved in Remark 3.2 of [FJ].

\section{REFERENCES}

[AXZ] R. AUlaskari, J. XIAO AND R. H. ZhaO, On subspaces and subsets of BMOA and UBC, Analysis 15 (1995) 101-121.

[CF] S.-Y. A. Chang AND R. Fefferman, A continuous version of duality of $H^{1}$ and BMO, Ann. of Math. 112 (1980), 179-201.

[DX] G. DAFNi AND J. XIAO, Some new tent spaces and duality theorems for fractional Carleson measures and $Q_{\alpha}\left(\boldsymbol{R}^{n}\right)$, J. Funct. Anal. 208 (2004), 377-422 .

[EJPX] M. Essén, S. JANSOn, L. PEng AND J. XIAO, Q spaces of several real variables, Indiana Univ. Math. J. 49 (2000), 575-615.

[EX] M. Essén And J. XIAO, Some results on $Q_{p}$ spaces, $0<p<1$, J. Reine Angew. Math. 485 (1997), 173-195.

[Fe] C. FefFerman, Characterizations of bounded mean oscillation, Bull. Amer. Math. Soc. 77 (1971), 587588.

[FeSt] C. Fefferman And E. M. Stein, $H^{p}$ spaces of several variables, Acta Math. 129 (1972), $137-193$.

[FJ] M. FrazIER AND B. JawerTh, Decomposition of Besov spaces, Indiana Univ. Math. J. 34 (1985), $777-$ 799.

[FJW] M. FraZIER, B. JaWERTh AND G. Weiss, Littlewood-Paley Theory and the Study of Function Spaces, CBMS Regional Conference Series in Math., 79, American Math. Society, 1991.

[GJ] J. Garnett And P. W. Jones, BMO from dyadic BMO, Pacific J. Math. 99 (1982), 351-371.

[Ja] S. JANSON, On the space $Q_{p}$ and its dyadic counterpart, Complex analysis and differential equations (Uppsala, 1997), 194-205, Acta Univ. Upsaliensis Skr. Uppsala Univ. C Organ. Hist. 64, Uppsala Univ., Uppsala 1999. 
[JN] F. John And L. Nirenberg, On functions of bounded mean oscillation, Comm. Pure Appl. Math. 14 (1961), 415-426.

[M] Y. MEYER, Wavelets and operators; Translated from the 1990 French original by D. H. Salinger, Cambridge Studies in Advanced Mathematics, vol. 37, Cambridge University Press, Cambridge, 1992.

[RS] R. Rochberg And S. Semmes, A decomposition theorem for BMO and applications, J. Funct. Anal. 67 (1986), 228-263.

[St] E. M. STEIN, Harmonic analysis: real-variable methods, orthogonality, and oscillatory integrals, Princeton Univ. Press, Princeton, New Jersey, 1993.

[U] A. Uchiyama, A constructive proof of the Fefferman-Stein decomposition of BMO $\left(\boldsymbol{R}^{n}\right)$, Acta Math. 148 (1982), 215-241.

[WX] Z. WU AND C. XIE, Decomposition theorems for $Q_{p}$ spaces, Ark. Mat. 40 (2002), 383-401.

DEPARTMENT OF MATHEMATICS AND STATISTICS

CONCORDIA UNIVERSITY

1455 de MAisonneuve Blvd. West

MONTRÉAL, QUÉBEC, H3G 1M8

CANADA

E-mail address: gdafni@mathstat.concordia.ca
DEPARTMENT OF MATHEMATICS AND STATISTICS MEMORIAL UNIVERSITY OF NEWFOUNDLAND ST. JOHN's, NL, A 1C 5S7

CANADA

E-mail address: jxiao@math.mun.ca 